\title{
Water-Dispersible Silica-Coated Upconverting Liposomes: Can a Thin Silica Layer Protect TTA-UC against Oxygen Quenching?
}

Sven H. C. Askes, ${ }^{\dagger}$ Vincent C. Leeuwenburgh, ${ }^{\dagger}$ Wim Pomp, ${ }^{\dagger}$ Hadi Arjmandi-Tash, ${ }^{\dagger}$ Stefania $^{\text {Tanase }}{ }^{\S}$ Thomas Schmidt, ${ }^{*}$ and Sylvestre Bonnet* ${ }^{*} \mathbb{E}^{0}$

${ }^{\dagger}$ Leiden Institute of Chemistry and ${ }^{\ddagger}$ Leiden Institute of Physics, Leiden University, 2300 RA Leiden, The Netherlands

${ }^{\S}$ Van 't Hoff Institute for Molecular Sciences, University of Amsterdam, 1090 GS Amsterdam, The Netherlands

Supporting Information

ABSTRACT: Light upconversion by triplet-triplet annihilation (TTA-UC) in nanoparticles has received considerable attention for bioimaging and light activation of prodrugs. However, the mechanism of TTA-UC is inherently sensitive for quenching by molecular oxygen. A potential oxygen protection strategy is the coating of TTA-UC nanoparticles with a layer of oxygenimpermeable material. In this work, we explore if (organo)silica can fulfill this protecting role. Three synthesis routes are described for preparing water-dispersible (organo)silica-coated red-to-blue upconverting liposomes. Their upconversion properties are investigated in solution and in A549 lung carcinoma cells. Although it was found that the silica offered no protection

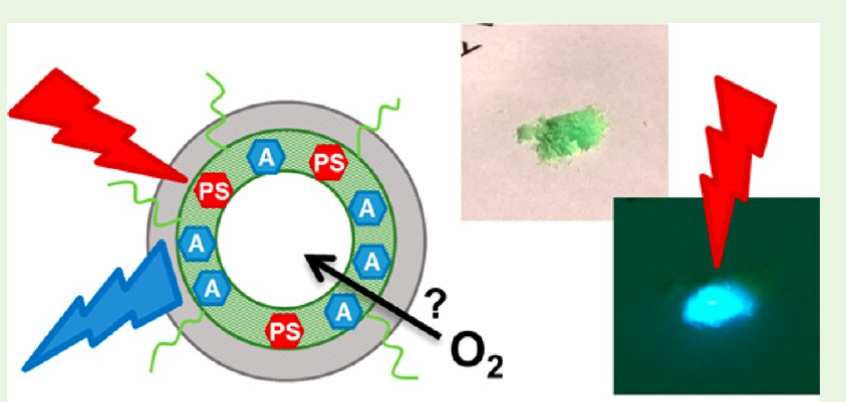

Silica-coated upconverting liposomes

from oxygen in solution and after uptake in A549 cancer cells, upon drying of the silica-coated liposome dispersion in an excess of (organo)silica precursor, interesting liposome-silica nanocomposite materials were obtained that were capable of generating blue light upon red light excitation in air.

KEYWORDS: liposomes, silica coating, nanoparticles, light upconversion, oxygen quenching, photonic materials

\section{INTRODUCTION}

Photon upconversion is the generation of high energy light from low energy light, for example, turning red light into blue light. Among the wide variety of applications, light upconversion has received substantial interest in upconversion bioimaging and as method to shift the activation wavelength of photoactivatable anticancer prodrugs toward the phototherapeutic window. ${ }^{1-6}$ One mechanism of light upconversion is triplet-triplet annihilation upconversion (TTA-UC), which is particularly attractive because it can be generated at low excitation power (even lower than $1 \mathrm{~mW} \mathrm{~cm}^{-2}$ ) 7 with relatively high efficiency ( $\sim 5 \%$ in aqueous nanoparticle systems)., TTA-UC is based on the photophysical interplay of photosensitizer and annihilator chromophores (see Figure S1). ${ }^{10-13}$ The photosensitizer absorbs low energy light, after which a long-lived triplet excited state is reached via intersystem crossing. The energy of this triplet state is transferred to the annihilator upon diffusional collision by means of triplet-triplet energy transfer (TTET); a succession of TTET leads to a buildup of long-lived triplet state annihilators. Two triplet state annihilators can then perform triplet-triplet annihilation upconversion, in which one of them departs with all the energy and reaches a high energy singlet excited state. Finally, this singlet excited state returns to the ground state by fluorescent emission of a high energy photon, realizing light upconversion. TTA-UC has been demonstrated in an extensive assortment of organic, inorganic, and/or supramolecular materials, ${ }^{14-19}$ as well as in nano- or microsized particles, ${ }^{20,21}$ and has been used for applications in photocatalysis, ${ }^{22,23}$ solar energy harvesting, ${ }^{24-27}$ drug delivery and activation, ${ }^{1,2}$ and bioimaging. ${ }^{8,9,28-32}$

Obviously, for biological application of TTA-UC supramolecular assemblies are required to colocalize photosensitizer and annihilator. Liposomes functionalized with PEGylated lipids have emerged over decades as supramolecular tools in drug delivery because of their high biocompatibility, low toxicity, and selective accumulation in tumors as a result of the enhanced permeability and retention effect (EPR)..$^{33-35}$ Our group recently combined liposomes with TTA-UC: red-to-blue upconversion was demonstrated in the lipid bilayer of neutral PEGylated DMPC (1,2-dimyristoyl-sn-glycero-3-phosphocholine) liposomes. ${ }^{1,2}$ However, upconversion could not be realized in air, because molecular oxygen readily quenches the triplet states of sensitizer and annihilator. In other words, the upconverting drug carrier did not function in oxygen-rich conditions, and the use of such systems in vivo would lead to unreliable performance, because oxygen concentrations vary drastically in biological tissues. ${ }^{36-38}$ The oxygen sensitivity of

Received: November 1, 2016

Accepted: January 17, 2017

Published: January 17, 2017 
TTA-UC can be reduced by developing upconversion systems that either (i) feature very fast TTA-UC so that upconversion takes place faster than physical quenching by molecular oxygen, ${ }^{7,17}$ (ii) have built-in chemical agents that consume molecular oxygen, ${ }^{8,39-41}$ or (iii) are protected by a physical barrier that cannot be crossed by molecular oxygen. Most noteworthy examples of the latter strategy include upconverting oil-core nanocapsules embedded in an oxygen protective cellulose material or poly(vinyl alcohol) nanofibers, ${ }^{19,42}$ and upconversion in hyperbrached unsaturated polyphosphoesters. ${ }^{43}$ However, there are no examples yet where a nanoscale oxygen-barrier is used to protect TTA-UC in a drug delivery system. In this work, we attempt to coat upconverting liposomes with silica or organosilica as potential oxygen barrier and investigate the oxygen protection potential of such a silica barrier.

Using silica as a barrier has several advantages. First of all, silica is recognized as a chemically inert, biocompatible, $\mathrm{pH}$ insensitive, and transparent material. ${ }^{44-47}$ Second, the silica surface can be modified to attach molecules such as PEG, biotin, or ruthenium(II) complexes. ${ }^{48}$ Finally, it has been demonstrated that nanometer-thick silica layers can act as an oxygen barrier in silica-coated polymer films, ${ }^{49}$ and it has been suggested that silica protects oxygen-sensitive chromophores such as $\left[\mathrm{Ru}(\mathrm{bpy})_{3}\right]^{2+}$ and $\left[\mathrm{Ru}(\mathrm{phen})_{3}\right]^{2+}$ in doped silica nanoparticles. ${ }^{50-52}$ Silica-coating of liposomes has been described before ${ }^{53-59}$ for example, Bégu et al. described the application of a silica-coating to DPPC liposomes (1,2dipalmitoyl-sn-glycero-3-phosphocholine) by sequential hydrolysis and condensation of tetraethylorthosilicate (TEOS) as silica precursor. ${ }^{53,54,60-62}$ It was suggested that the deposition of silica on the membrane was controlled by hydrogen-bonding interactions between the phosphate groups of the lipids, interfacial water, and silanol groups of the silica. Furthermore, nitrogen adsorption isotherms suggested that the dried particles were nonporous. However, most of the published articles do not explicitly discuss whether these particles can be redispersed in aqueous buffers and how stable their dispersion are, which are two critical criteria for drug delivery systems.

This work describes three synthetic routes for obtaining water-dispersible (organo)silica-coated DMPC liposomes containing a red-to-blue upconverting couple, i.e. palladium tetraphenyltetrabenzoporphyrin (PdTPTBP) and perylene (Figure 1). In a second step, the upconverting properties of silica-coated liposomes are investigated in order to assess
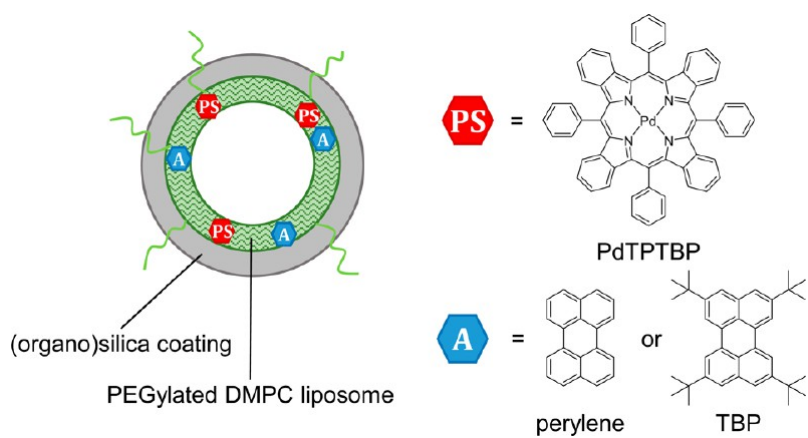

Figure 1. Schematic representation of (organo)silica-coated liposomes containing photosensitizer (PS; PdTPTBP) and annihilator chromophores $(\mathrm{A}$; perylene or TBP $)$. PdTPTBP = palladium tetraphenyltetrabenzoporphyrin, TBP $=3,5,8,11$-tetra (tert-butyl)perylene. whether silica can act as an oxygen-barrier to allow upconversion in air. Furthermore, the uptake of these particles by cancer cells and their ability to perform upconversion in vitro will be evaluated. Finally, the silica-coated liposomes were dried in the presence of an excess of (organo)silica precursor and the upconversion properties of the resulting nanocomposite materials are investigated.

\section{MATERIALS AND METHODS}

General. 1,2-Dilaureyl-sn-glycero-3-phosphocholine (DLPC), 1,2dimyristoyl-sn-glycero-3-phospho choline (DMPC), 1,2-dipalmitoylsn-glycero-3-phosphocholine (DPPC), and sodium $\mathrm{N}$-(carbonylmethoxypolyethylene glycol-2000)-1,2-distearoyl-sn-glycero-3-phosphoethanolamine (DSPE-mPEG-2000) were purchased from Lipoid $\mathrm{GmbH}$ (Ludwigshafen, Germany) and stored at $-18{ }^{\circ} \mathrm{C}$. Palladium tetraphenyltetrabenzoporphyrin (PdTPTBP) was purchased from BioConnect (Huissen, The Netherlands). Perylene was purchased from Sigma-Aldrich Chemie BV (Zwijndrecht, The Netherlands). The synthesis of 2,5,8,11-tetra(tert-butyl)perylene (TBPe) is described elsewhere. ${ }^{63}$ Dulbecco's phosphate buffered saline (DPBS) was purchased from Sigma-Aldrich and had a formulation of $8 \mathrm{~g} \mathrm{~L}^{-1}$ $\mathrm{NaCl}, 0.2 \mathrm{~g} \mathrm{~L}^{-1} \mathrm{KCl}, 0.2 \mathrm{~g} \mathrm{~L}^{-1} \mathrm{KH}_{2} \mathrm{PO}_{4}$, and $1.15 \mathrm{~g} \mathrm{~L}^{-1} \mathrm{~K}_{2} \mathrm{HPO}_{4}$ with a $\mathrm{pH}$ of 7.1-7.5. All other chemicals were obtained from the major companies and used as received.

Instrumentation. Ultracentrifugation was done with a BeckmanCoulter Optima L-90K ultracentrifuge, equipped with a $70.1 \mathrm{Ti}$ rotor, at $50 \mathrm{krpm}(230000 \mathrm{~g})$ for $30 \mathrm{~min}$. Freeze-dried samples were prepared with a Christ Alpha 1-2 LDPlus machine, operating at $<0.03$ mbar. Liposome or silica-coated liposome samples were placed in 50 $\mathrm{mL}$ round-bottom flasks, frozen in liquid nitrogen while gently swirling, and attached to the freeze-dryer. Dynamic Light Scattering (DLS) measurements were performed on undiluted samples $([$ DMPC $]=10 \mathrm{mM})$ using a Zetasizer nano $\mathrm{S}$ (Malvern Instruments) operating at $633 \mathrm{~nm}$, with 3 measurements of 12 runs each time. Zetapotential measurements were performed on a Zetasizer nano ZS (Malvern Instruments), at $25{ }^{\circ} \mathrm{C}$ with 3 measurements and $10-100$ automatic runs. Samples were diluted 20X in Milli-Q in a DTS1070 cell, at a known $\mathrm{pH}$, so that $[\mathrm{DMPC}]=0.5 \mathrm{mM}$. Fourier Transform Infrared (FT-IR) spectra were recorded on a PerkinElmer Paragon 1000. Transmission Electron Microscopy (TEM) imaging was performed on a JEOL 1010 TEM using accelerating voltages of 60.0 or $80.0 \mathrm{kV}$, iTEM software and a Olympus Megaview G2 camera. Samples were loaded onto Formvar-coated carbon grids (Van Loenen instruments, Netherlands) by depositing a grid on top of a sample droplet for about $30 \mathrm{~min}$. CP-MAS ${ }^{29} \mathrm{Si}$ nuclear magnetic resonance (NMR) spectroscopy was performed on a Bruker AV400 using a relaxation delay of $60 \mathrm{~s}$ and pulse duration of $3 \mu \mathrm{s}$. Scanning Electron Microscopy (SEM) was performed on a Nova NanoSEM (FEI) using accelerating voltages of $15.0 \mathrm{kV}$. Powder samples were deposited on conducting tape. Thermogravimetric Analysis (TGA) measurements were performed on a Netzsch STA with a DSC/TG $\mathrm{Al}_{2} \mathrm{O}_{3}$ pan crucible, with a temperature increasing from 30 to $500{ }^{\circ} \mathrm{C}$ at $10{ }^{\circ} \mathrm{C}$ $\mathrm{min}^{-1}$, and a gas flow of $40 \mathrm{~mL} \mathrm{~min}{ }^{-1}$.

Preparation of Upconverting Liposomes. Aliquots of stock solutions in chloroform were added together in a round-bottom flask to obtain a mixture of DMPC lipid $\left(5 \mathrm{mM}\right.$ in $\mathrm{CHCl}_{3}, 10 \mathrm{~mL}, 50$ $\mu \mathrm{mol})$, DSPE-mPEG-2000 (0.2 mM in $\left.\mathrm{CHCl}_{3}, 10 \mathrm{~mL}, 2 \mu \mathrm{mol}\right)$, PdTPTBP $\left(10 \mu \mathrm{M}\right.$ in $\left.\mathrm{CHCl}_{3}, 2.5 \mathrm{~mL}, 25 \mathrm{nmol}\right)$ and perylene $(0.2 \mathrm{mM}$ in $\left.\mathrm{CHCl}_{3}, 1.25 \mathrm{~mL}, 250 \mathrm{nmol}\right)$. For liposomes used in cell treatment, the perylene dye was replaced by TBPe in the same amount. The solvent was removed by rotary evaporation at $50{ }^{\circ} \mathrm{C}$ under reduced pressure and then under high vacuum for at least $15 \mathrm{~min}$. PBS buffer ( $5 \mathrm{~mL}$ ) was added to the lipid film to obtain a final DMPC lipid concentration of $10 \mathrm{mM}$. The flask was then freeze-thawed using liquid nitrogen and a water bath at $50{ }^{\circ} \mathrm{C}$ for 3 cycles, and the suspension was subsequently extruded using a $200 \mathrm{~nm}$ Nuclepore polycarbonate filter and a mini-extruder (Avanti Polar Lipids, Inc.) at $55{ }^{\circ} \mathrm{C}$, for at least 11 times. All dyes were incorporated into the liposomes with minimal losses, as indicated by the lack of color on the 
filter after the extrusion. The resulting liposome suspension was analyzed by DLS before use in further synthesis steps.

Silica-Coating of Upconverting Liposomes-Method A. According to modified literature procedures. ${ }^{53,60,61}$ (3-Aminopropyl)triethoxysilane (APTES, $293 \mu \mathrm{L}, 1.25 \mathrm{mmol}$ ) was added to the liposome solution (prepared as mentioned above, $5 \mathrm{~mL}$, [DMPC] $\approx 5$ $\mathrm{mM}$ ) and the mixture was stirred for $16 \mathrm{~h}$. At this point, the $\mathrm{pH}$ was 10.7. To remove unreacted and unassociated APTES, the sample was ultracentrifuged and resuspended in $5 \mathrm{~mL}$ PBS twice, which neutralized the $\mathrm{pH}$. This washing procedure did not affect the particle size distribution and shape, as judged by TEM (data not shown). Meanwhile, tetraethylorthosilicate (TEOS) was prehydrolyzed in PBS (typically $50 \mathrm{mM}$ TEOS) for $24 \mathrm{~h}$ at $40^{\circ} \mathrm{C}$, creating a solution of 50 $\mathrm{mM}$ prehydrolyzed TEOS called "preTEOS". Preliminary experiments determined that a prehydrolysis time of $24 \mathrm{~h}$ was optimal for $50 \mathrm{mM}$ TEOS in PBS. A longer time resulted in the formation of nondesired silica nanoparticles (observed by DLS), and a shorter time resulted in sample aggregation during liposome coating. Higher TEOS concentrations resulted in formation of silica nanoparticles as well. Thus, 8 $\mathrm{mL}$ preTEOS $(50 \mathrm{mM}, 400 \mu \mathrm{mol})$ was added to the APTES-coated liposome suspension $(5 \mathrm{~mL})$ and the mixture was stirred for $24 \mathrm{~h}$ at 20 ${ }^{\circ} \mathrm{C}$. The final APTES-preTEOS-coated liposomes were purified by ultracentrifugation and redispersion in $5 \mathrm{~mL}$ of Milli-Q or PBS (once).

Silica-Coating of Upconverting Liposomes-Method B. Liposomes were prepared as mentioned above, but instead of PBS, $1 \mathrm{M} \mathrm{HCl}$ in PBS was used to hydrate the lipid film. The liposome assembly under such acidic conditions produced high quality liposomes (z-ave $134 \mathrm{~nm}, 0.1 \mathrm{PDI})$. After liposome assembly, TEOS (36 $\mu \mathrm{L}, 160 \mu \mathrm{mol}$ ) was added to $2 \mathrm{~mL}$ of the liposome solution $([\mathrm{DMPC}] \approx 5 \mathrm{mM})$ and stirred for $30 \mathrm{~min}$. Then, the solution was transferred to a dialysis bag (Servapor, MW cutoff 12-14 kDa; SERVA Electrophoresis $\mathrm{GmbH}$ ) and dialyzed against demineralized water (1 L) for $24 \mathrm{~h}$, during which time the water was refreshed twice.

Silica-Coating of Upconverting Liposomes-Method C. First, TEOS was prehydrolyzed in PBS (typically $50 \mathrm{mM}$ TEOS) for $24 \mathrm{~h}$ at $40{ }^{\circ} \mathrm{C}$, creating a solution of $50 \mathrm{mM}$ prehydrolyzed TEOS called "preTEOS". Eight mL PreTEOS $(400 \mu \mathrm{mol})$ was then added to the liposome suspension (prepared as above, $5 \mathrm{~mL},[\mathrm{DMPC}] \approx 5 \mathrm{mM}$ ) and stirred for $24 \mathrm{~h}$ at $20^{\circ} \mathrm{C}$. These TEOS-coated liposomes were ultracentrifuged and redispersed in $5 \mathrm{~mL}$ PBS twice to remove unreacted and unassociated TEOS. APTES ( $293 \mu \mathrm{L}, 1.25 \mathrm{mmol})$ was added to the coated liposome solution $(5 \mathrm{~mL})$ and the solution was stirred overnight for $16 \mathrm{~h}$. The final preTEOS-APTES-coated liposomes were purified by ultracentrifugation and redispersion in 5 $\mathrm{mL}$ Milli-Q or PBS (once).

Preparation of (Silica-Coated) Liposome Solids. Freeze-dried liposome solids were prepared by freeze-drying at $\leq 0.03 \mathrm{mbar}$ (the instrumentation section). Oven-dried silica-coated liposome solids were prepared by depositing unpurified silica-coated liposomes A-UL or ApT-UL (i.e., including excess APTES and/or preTEOS) in $5 \mathrm{~mL}$ portions on watch glasses and drying overnight at $50{ }^{\circ} \mathrm{C}$.

UV-Vis Absorption and Emission Spectroscopy. Absorption and emission spectroscopy was performed with a custom-built setup (Figure S11) All optical parts were connected with FC-UVxxx-2 (xxx $=200,400,600$ ) optical fibers from Avantes (Apeldoorn, The Netherlands), with a diameter of $200-600 \mu \mathrm{m}$, respectively, and that were suitable for the UV-vis range $(200-800 \mathrm{~nm})$. Typically, a 2 $\mathrm{mL}$ sample was transferred in a 111-OS macro fluorescence cuvette from Hellma and placed in a CUV-UV/vis-TC temperature-controlled cuvette holder from Avantes (Apeldoorn, The Netherlands). Every time the temperature was changed, the sample was allowed to equilibrate for $5 \mathrm{~min}$. For emission spectroscopy, the samples were irradiated from the side with a $10 \mathrm{~mW} 630 \mathrm{~nm}$ laser light beam from a clinical grade Diomed $630 \mathrm{~nm}$ PDT laser $\left(4 \mathrm{~mm}\right.$ beam, $80 \mathrm{~mW} \mathrm{~cm}{ }^{-2}$ ). The $630 \mathrm{~nm}$ light was filtered through a FB630-10, $630 \mathrm{~nm}$ band-pass filter (Thorlabs, Dachau/Munich, Germany) put between the laser and the sample. The excitation power was controlled using a NDL-25C-4 variable neutral density filter (Thorlabs), and measured using a S310C thermal sensor connected to a PM100USB power meter (Thorlabs). An Avantes 2048L StarLine CCD spectrometer was connected at $90^{\circ}$ angle with respect to the excitation source. A Thorlabs NF-633 notch filter placed between the cuvette holder and the spectrometer was used to block the excitation light. To make the emission spectra of the different samples in solution comparable, the samples were diluted in PBS so that $A_{630}=0.20$. Optionally, $\mathrm{Na}_{2} \mathrm{SO}_{3}(1 \mathrm{~mL}, 100 \mathrm{mM}$ in PBS, $\mathrm{pH}$ 7.4) was freshly added to $1 \mathrm{~mL}$ samples so that $50 \mathrm{mM} \mathrm{Na}_{2} \mathrm{SO}_{3}$ was present for oxygen scavenging during spectroscopy. UV-vis absorption spectra were measured using an Avalight-DHc halogendeuterium lamp (Avantes) as light source and a 2048L StarLine spectrometer (Avantes) as detector, both connected to the cuvette holder at a $180^{\circ}$ angle and both at a $90^{\circ}$ angle with respect to the red laser irradiation direction. The filter holder between cuvette holder and detector was in a position without a filter. Luminescence emission spectra were measured using the same detector but with the UV-vis light source switched off. All spectra were recorded with Avasoft software from Avantes and further processed with Microsoft Office Excel 2010 and Origin Pro software.

Solid-State Emission Spectroscopy. Solid state emission spectroscopy was done in a slightly different setup than for liquid samples (Figure S12). A bifurcated fiber (FCB UVIR 400-2, Avantes) was connected to the top of the cuvette holder, in which a lens (Avantes COL-UV/vis lens, $f=8.7 \mathrm{~mm}$ ) was fitted that simultaneously transmitted excitation light and captured the emission and $7.1 \mathrm{mg}$ of solid sample was deposited on the bottom of a semimicro cuvette. Samples were irradiated with $30 \mathrm{~mW} 630 \mathrm{~nm}$ light $(2.4 \mathrm{~mm}$ beam, $\left.0.66 \mathrm{~W} \mathrm{~cm}^{-2}\right)$.

General Cell Culturing. A549 human lung carcinoma cells were cultured in $25 \mathrm{~cm}^{2}$ flasks in $8 \mathrm{~mL}$ Dulbecco's modified Eagle medium with phenol red (DMEM; Sigma Life Science, USA), supplemented with $8.2 \% \mathrm{v} / \mathrm{v}$ fetal calf serum (FCS; Hyclone), $200 \mathrm{mg} \cdot \mathrm{L}^{-1}$ penicillin and streptomycin $(\mathrm{P} / \mathrm{S}$; Duchefa), and $1.8 \mathrm{mM}$ glutamine $\mathrm{S}$ (GM; Gibco, USA), under standard culturing conditions (humidified, $37{ }^{\circ} \mathrm{C}$ atmosphere containing $7.0 \% \mathrm{CO}_{2}$ ). The cells were split approximately once per week upon reaching $70-80 \%$ confluency, using seeding densities of $2 \times 10^{5}$ cells, and the medium was refreshed once per week. Cells were passaged for 4-8 weeks.

Regular Fluorescence Microscopy. For regular fluorescence microscopy experiments, cells were seeded into 6-well plates, $200 \mathrm{~K}$ cells per well. Meanwhile, the liposome- or silica-coated liposome samples at a $2.5 \mathrm{mM}$ lipid concentration were filtered through a 0.45 $\mu \mathrm{m}$ filter and further brought to a $1 \mathrm{mM}$ final lipid concentration with OptiMEM (Life Technologies, USA), supplemented with 2.5\% FCS, $200 \mathrm{mg} / \mathrm{L} \mathrm{P} / \mathrm{S}$, and $1.8 \mathrm{mM}$ GM ("OptiMEM complete"). Twentyfour hours after cell seeding, $3 \mathrm{~mL}$ of liposome mixture was added to each well, and the cells were incubated for another $24 \mathrm{~h}$. The liposomes were removed and the cells were washed once with PBS and supplied with $1 \mathrm{~mL}$ of OptiMEM complete. The cells were imaged in bright-field mode ( $250 \mathrm{~ms}$ exposure) and with $377 \mathrm{~nm}$ excitation (1000 ms exposure) using a Leica SPE confocal microscope at 20x magnification and Cell $\wedge \mathrm{M}$ software.

Upconversion Luminescence Microscopy. For upconversion microscopy experiments, cells were seeded at a density of 30000 cells per well on $25 \mathrm{~mm}$ diameter microscopy coverslips (VWR, thickness no. 1) in 6-well plates. Meanwhile, the liposome- or silica-coated liposome-samples at a $2.5 \mathrm{mM}$ lipid concentration were filtered through a $0.45 \mu \mathrm{m}$ pore filter and further brought to a $1 \mathrm{mM}$ final lipid concentration with OptiMEM complete. Twenty-four hours after seeding, $3 \mathrm{~mL}$ of liposome-medium mixture was added to each well and incubated for $24 \mathrm{~h}$. The liposomes were then washed once with PBS and supplied with $1 \mathrm{~mL}$ of OptiMEM complete. The coverslips were transferred to custom-made coverslip holders, which in turn were put in a stage-top miniature incubator (Tokai Hit, INUBG2ETFPWSKM) fitted with a GM- 8000 gas controller. The cells were incubated for $30 \mathrm{~min}$ at $1 \% \mathrm{O}_{2}, 7 \% \mathrm{CO}_{2}$, and $37^{\circ} \mathrm{C}$ before imaging. Imaging was performed with a customized Zeiss Axiovert S100 Inverted Microscope setup, fitted with a Zeiss 100x Plan Apochromat 1.4 NA oil objective, and an Orca Flash 4.0 V2 sCMOS camera from Hamamatsu, which together produced images with pixel size of $69 \mathrm{~nm}$ (for $100 \times$ ). The typical camera exposure time was $1000 \mathrm{~ms}$. Excitation at $405 \mathrm{~nm}$ was performed with a CrystaLaser DL405-050 diode laser, 
in combination with a Chroma zet442/514/568m emission filter and Chroma zt405/514/561rpc dichroic mirror. The output power of the $405 \mathrm{~nm}$ laser at the sample was typically $62 \mu \mathrm{W}$ at $100 \times$ magnification $\left(60 \mu \mathrm{m}\right.$ spot diameter, intensity 2.2 W.cm $\left.{ }^{-2}\right)$. Excitation at $639 \mathrm{~nm}$ was performed with a Power Technology 1Q1A30(639-35B)G3 diode laser, in combination with a $575 \mathrm{~nm}$ short pass filter (Edmund Optics, part no. \#84-709) and Chroma zt405/532/635rpc dichroic mirror. The output power of the $639 \mathrm{~nm}$ laser at the sample was typically $1.0 \mathrm{~mW}$ at $100 \times$ magnification $(70 \mu \mathrm{m}$ spot diameter, $26 \mathrm{~W}$ $\mathrm{cm}^{-2}$ intensity).

\section{RESULTS AND DISCUSSION}

Preparation and Characterization of Upconverting Liposomes. First, upconverting DMPC liposomes (codenamed UL) were prepared according to a literature procedure. ${ }^{1}$ Briefly, a mixture of $5 \mathrm{mM}$ 1,2-dimyristoyl-sn-glycero-3phosphocholine (DMPC), $4 \mathrm{~mol} \%$ sodium $\mathrm{N}$-(carbonylmethoxypolyethylene glycol-2000)-1,2-distearoyl-sn-glycero-3phosphoethanolamine (DSPE-mPEG-2000) was used to prepare liposomes via a hydration-extrusion method in phosphate buffered saline (PBS). A red-to-blue upconverting TTA-UC couple was selected for incorporation in the liposomes, consisting of palladium(II) tetraphenyltetrabenzoporphyrin (PdTPTBP, $0.05 \mathrm{~mol} \%$ ) as red light photosensitizer, and perylene as blue emitter $(0.5 \mathrm{~mol} \%)$. Dynamic light scattering (DLS) measurements reported a reproducible average hydrodynamic diameter (z-ave) of around $150 \mathrm{~nm}$ and a polydispersity index (PDI) of 0.1 (Table 1). The UV-vis

Table 1. Physical Characterization of Liposomes UL and (Organo)silica-Coated Liposomes A-UL, pT-UL, pTA-UL, ApT-UL, and T-UL by Dynamic Light Scattering (DLS) and Zeta-Potentiometry, with Reported Average Hydrodynamic Diameter ( $z$-ave), Polydispersity Index (PDI), and Zeta Potential at the Given $\mathrm{pH}$

\begin{tabular}{lcccc}
\multicolumn{1}{c}{ sample } & $\begin{array}{c}z \text {-ave } \\
(\mathrm{nm})^{a}\end{array}$ & PDI $^{a}$ & $\begin{array}{c}\text { zeta potential } \\
(\mathrm{mV})\end{array}$ & $\mathrm{pH}$ \\
UL & $148 \pm 4$ & $0.09 \pm 0.01$ & $-16 \pm 0$ & 7.1 \\
A-UL (unwashed) & $171 \pm 5$ & $0.14 \pm 0.02$ & & \\
A-UL & $128 \pm 1$ & $0.10 \pm 0.02$ & $-40 \pm 5$ & 6.8 \\
ApT-UL & $145 \pm 1$ & $0.15 \pm 0.01$ & $-20 \pm 0$ & 7.0 \\
pT-UL & & & $-19 \pm 1$ & 6.9 \\
pTA-UL & $167 \pm 1$ & $0.15 \pm 0.01$ & $-17 \pm 1$ & 7.2 \\
T-UL & & & $-33 \pm 1$ & 6.7
\end{tabular}

${ }^{a}$ Standard deviation based on 3 or more individually prepared samples.

${ }^{b}$ Standard deviation based on 3 measurements of the same sample.

absorption spectrum (Figure 3) of UL shows the characteristic absorption peaks of perylene $(390,414,440 \mathrm{~nm})$ and PdTPTBP $(440,630 \mathrm{~nm})$. The emission spectrum $\left(\lambda_{\mathrm{exc}}=\right.$ $630 \mathrm{~nm}, 80 \mathrm{~mW} \cdot \mathrm{cm}^{-2}$ ) of UL in $50 \mathrm{mM}$ sodium sulfite in aerated PBS shows both the phosphorescence of PdTPTBP $(800 \mathrm{~nm})$ and the perylene-based emission $(473 \mathrm{~nm})$, characteristic of upconversion with this dye couple (Figure 3a). ${ }^{1}$ Figure $3 \mathrm{~b}$ shows the temperature dependence of the upconversion emission and phosphorescence. The upconversion first increases up to $25{ }^{\circ} \mathrm{C}$, and then decreases slightly, whereas the phosphorescence decreases steeply up to $25{ }^{\circ} \mathrm{C}$, and then continues to decrease, but less steeply. The rise in upconversion up to $25{ }^{\circ} \mathrm{C}$ is explained by the fact that the DMPC membrane undergoes a phase transition from a gel to a liquid crystalline phase at $24{ }^{\circ} \mathrm{C}$. ${ }^{64}$ In the liquid crystalline phase, the fluidity of the membrane is increased, and collision-

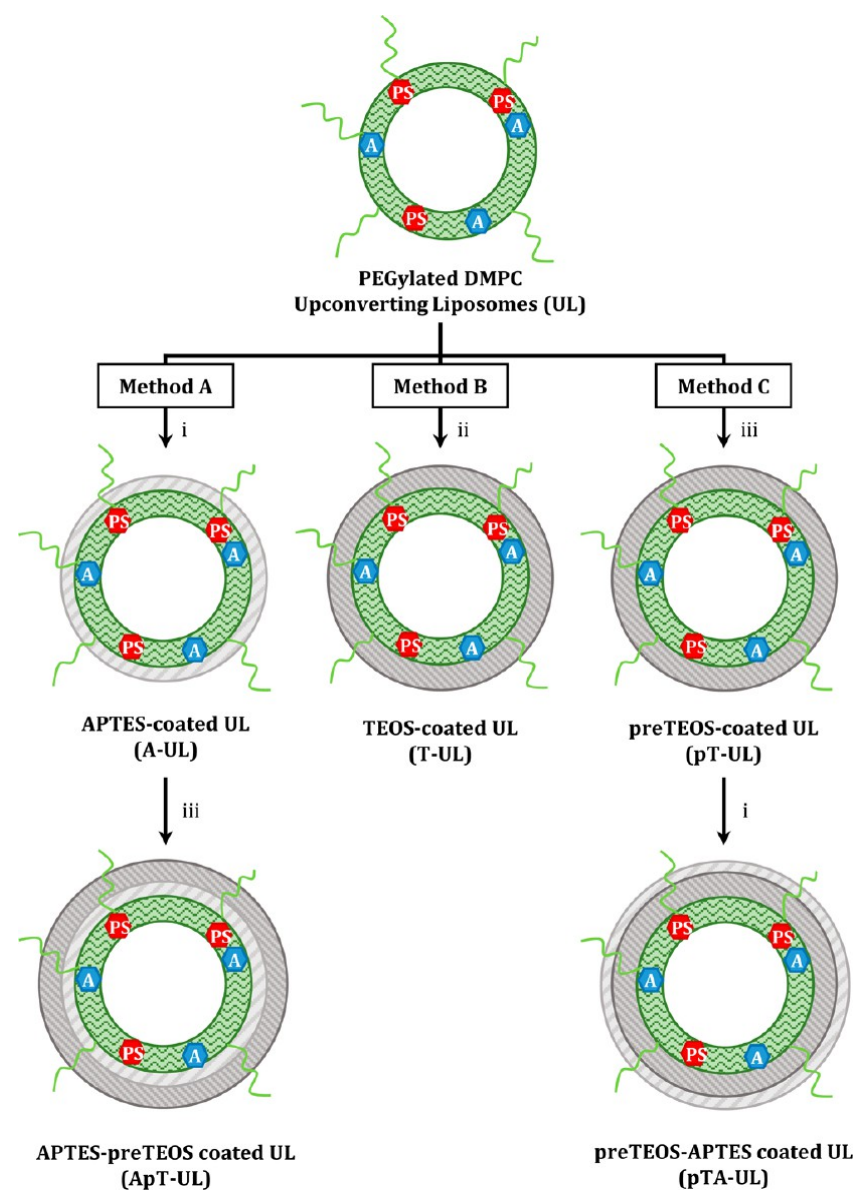

Figure 2. Three different synthesis methods to obtain silica-coated upconverting PEGylated liposomes. Conditions per eq DMPC: (i) 25 equiv. of APTES, $16 \mathrm{~h}$; (ii) 8 equiv. of TEOS, $1 \mathrm{M} \mathrm{HCl}, 30 \mathrm{~min}$ (iii) 8 equiv. of "preTEOS", $24 \mathrm{~h}$; preTEOS = TEOS, stirred for $24 \mathrm{~h}$ in PBS $(50 \mathrm{mM})$ at $40{ }^{\circ} \mathrm{C}$ before addition.

dependent processes such as TTET and TTA become more efficient, leading to more upconversion. ${ }^{1} \mathrm{~A}$ detailed discussion of this phenomenon is described in a separate communication. ${ }^{65}$ Below, the temperature-variation of the upconverted light intensity, which is a direct sign of the phase change of the membrane, and thus of the existence of an intact lipid bilayer, will be used to verify the integrity of the lipid bilayer after silicacoating.

Once the liposomes were prepared and characterized, silica coating was realized. Unfortunately, all our attempts to reproduce the work of Bégu et al., who described the synthesis of silica-coated liposomes based on direct addition of tetraethylorthosilicate (TEOS) to non-PEGylated DPPC liposomes, were unsuccessful. ${ }^{54}$ In our hands, these experiments inevitably led to the formation of silica nanoparticles, gelation of the reaction mixture, and/or aggregation of the silica-coated liposomes. Also, silica-coating experiments with 1,2-dilauroylsn-glycero-3-phosphocholine (DLPC) liposomes were unsuccessful. Therefore, only DMPC-based liposomes were further considered for preparing silica-coated liposomes. Three synthetic routes, called methods A, B, and C, were developed to apply an (organo)silica-coating method to upconverting DMPC liposomes and obtain monodispersed particles (Figure 2).

Preparation of Silica-Coated Liposomes-Method A. UL were subsequently used for silica-coating experiments. In 

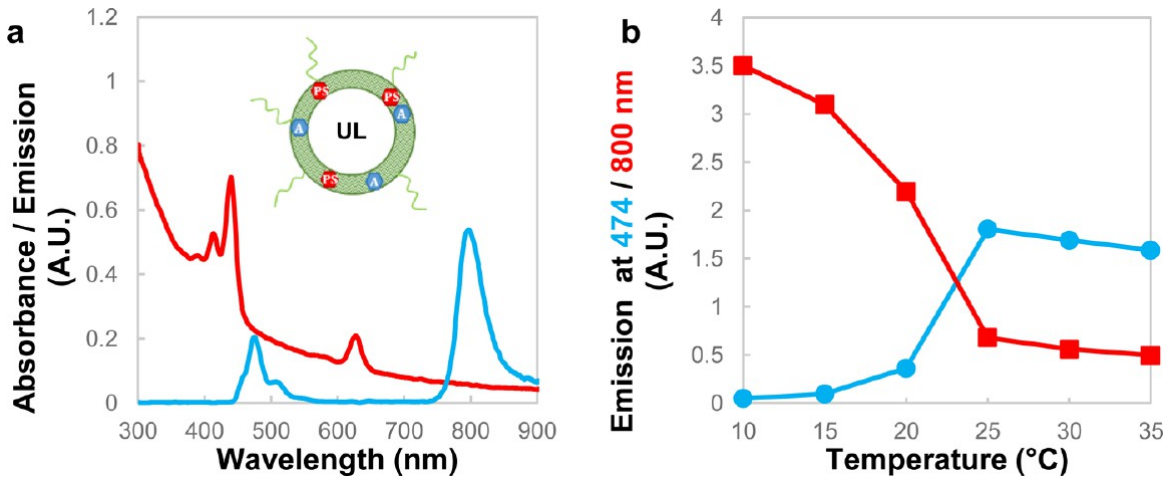

Figure 3. (a) Absorption spectrum (red) and emission spectrum (blue) of UL under $10 \mathrm{~mW} 630 \mathrm{~nm}$ irradiation $\left(80 \mathrm{~mW} \mathrm{~cm}^{-2}\right)$ at $20{ }^{\circ} \mathrm{C}$ in $50 \mathrm{mM}$ $\mathrm{Na}_{2} \mathrm{SO}_{3}$ PBS in air. (b) Temperature dependency of upconversion emission (at $474 \mathrm{~nm}$ ) and phosphorescence (at $800 \mathrm{~nm}$ ) of UL in $50 \mathrm{mM}$ $\mathrm{Na}_{2} \mathrm{SO}_{3} \mathrm{PBS}$ in air.

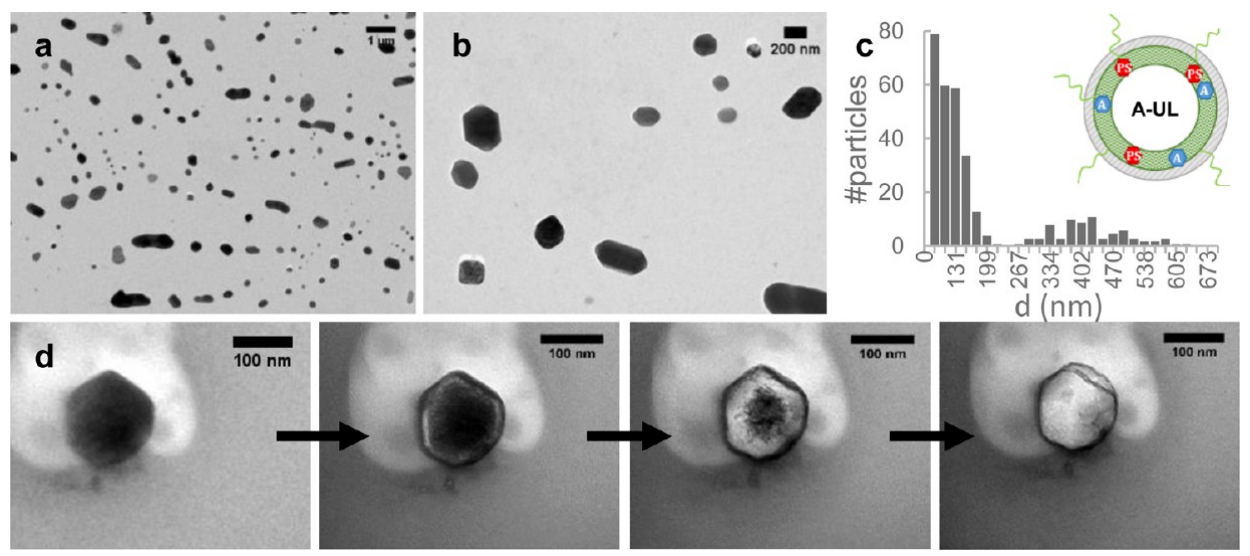

Figure 4. (a, b) TEM micrographs and (c) particle diameter distribution ( $N=324$ particles) of A-UL. (d) A-UL particle dries out over time and leaves behind an organosilica shell.

the first silica-coating method (method A), UL were first coated with (3-aminopropyl)triethoxysilane (APTES) to make organosilica-coated liposomes named A-UL, followed by additional coating with prehydrolyzed TEOS, making ApT-UL. APTES was chosen as initial layer because it has been suggested in the literature that the protonated amino group of APTES $\left(\mathrm{p} K_{\mathrm{a}}=\right.$ 10.4) associates with the negatively charged phospholipid head groups of the liposome membrane; ${ }^{56,57,66}$ in other words, the liposome membrane acts as a template on which APTES hydrolyses and condenses. After the first reaction step and before purification, DLS measurements showed an increase of $20 \mathrm{~nm}$ in hydrodynamic size of A-UL with respect to UL (Table 1), suggesting the deposition of an organosilica layer on the membrane of about $10 \mathrm{~nm}$ in thickness. As a control, UL were kept in the same reaction conditions, without adding APTES. The DLS values of UL remained unchanged during these $16 \mathrm{~h}$, which excludes that this change in size was caused by instability of the UL. Note that in the absence of the liposomes, APTES is likely to form five- or six-membered organosilicate rings in aqueous solution, which suppresses nanoparticle formation. ${ }^{67,68}$ Therefore, APTES alone cannot result in changes in DLS measurements.

To visually confirm the deposition of an APTES layer, A-UL were imaged by transmission electron microscopy (TEM), see Figure 4. The micrographs show individual particles in various polygonal shapes. No other nanoparticles were observed. Interestingly, when these particles were irradiated by an intense electron beam in the vacuum of the TEM, the liquid inside the particles visibly boiled and leaked out of the particles, leaving behind electron dense shells (Figure 4d). This observation suggested that the particles indeed consist of organosilicacoated liposomes. Surprisingly, the particles collapsed only upon high electron irradiation and the particles withstood the high vacuum of the TEM $\left(\sim 1 \times 10^{-5}\right.$ bar $)$ at low irradiance, which is evidence that the organosilica layer greatly fortifies the outer shell of a liposome. The average particle diameter from TEM (176 nm, Figure 4c) is consistent with the hydrodynamic size observed by DLS (171 nm, Table 1). The observed particles with a diameter of around $400 \mathrm{~nm}$ are likely to have been individual particles that merged during drying of the TEM grid, because these were absent in DLS measurements. Zeta potentiometry on A-UL gave a zeta-potential of $-30 \mathrm{mV}$ at $\mathrm{pH}$ 6.8 (Table 1). Such a negative surface charge was unexpected, given that the amino-groups of the organosilica layer are likely to be protonated at neutral $\mathrm{pH}$.

The second synthesis step, to make ApT-UL, involved coating of A-UL with prehydrolyzed TEOS, i.e., TEOS that had been hydrolyzed for $24 \mathrm{~h}$ prior to addition. ${ }^{60}$ This prehydrolysis step was found to be essential in acquiring monodispersed silica-coated liposomes: instead of hydrophobic TEOS, that may enter the liposome membrane and disrupt its structure, hydrolyzed TEOS (i.e., $\mathrm{Si}(\mathrm{OH})_{4}$ and small condensed oligomers) only condenses in solution. Without prehydrolysis the silica-coated liposomes aggregated quickly during the application of the coating. When TEOS was prehydrolyzed before addition to A-UL, DLS measurement of ApT-UL 


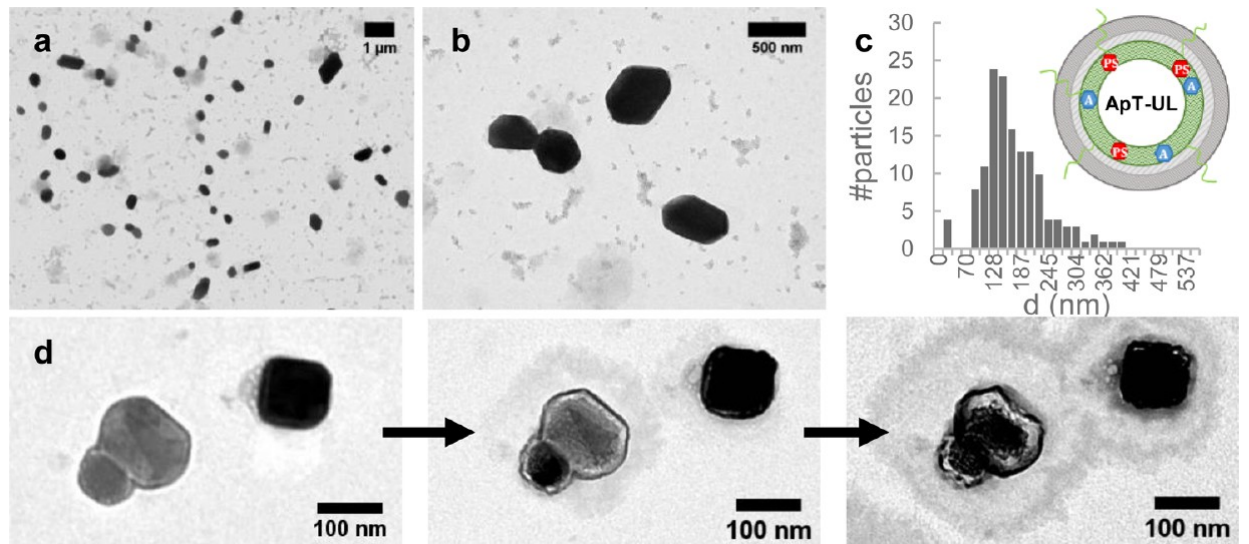

Figure 5. (a, b) TEM micrographs and (c) particle diameter distribution ( $N=142$ particles) of ApT-UL. (d) ApT-UL particles dry out and leave behind an (organo)silica shell. Note the leaking of salty water from the particles, leaving a stain around the particles. Gray vs black particles are due to differences in orientation and/or thicknesses.
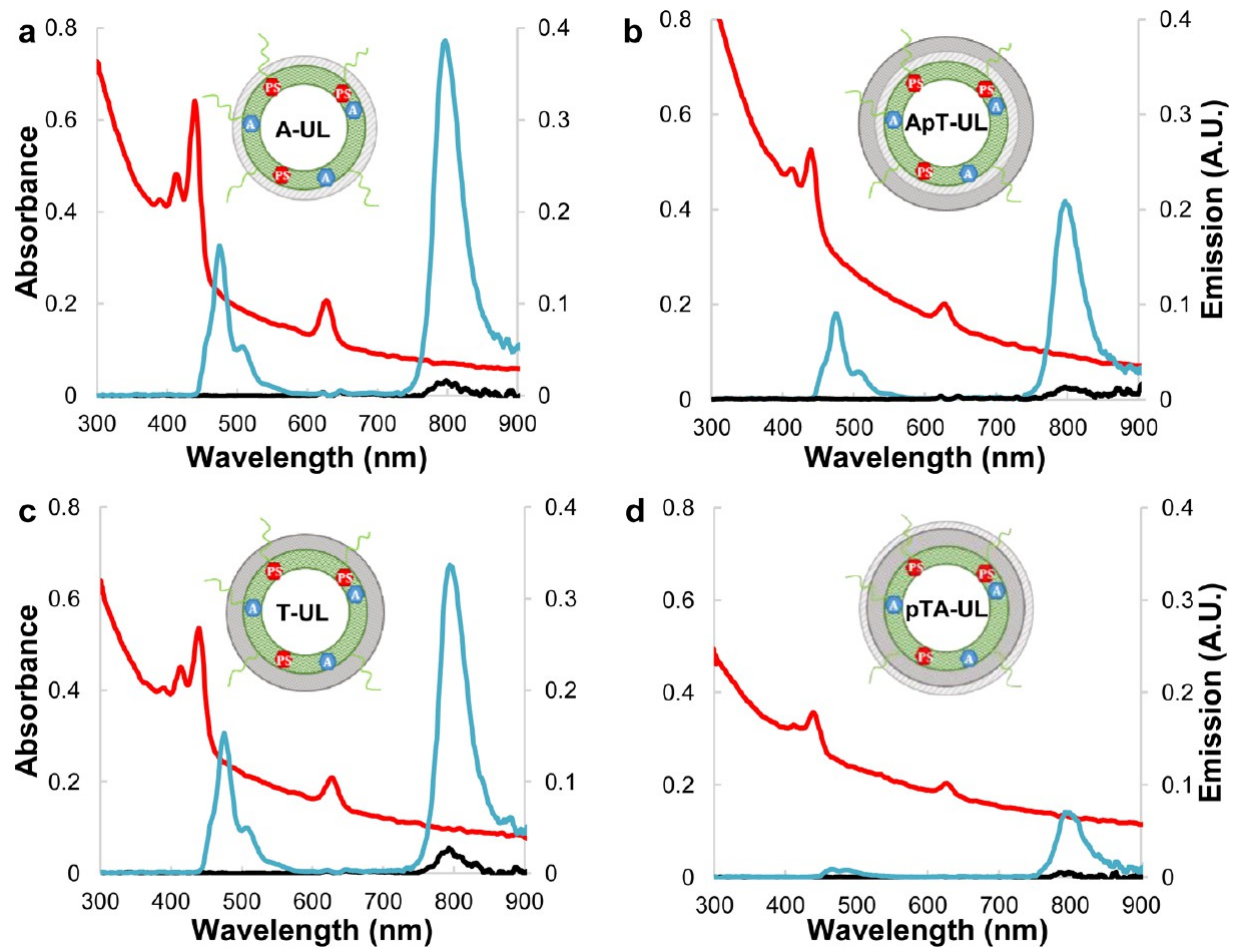

Figure 6. Absorption (red, left axes), and emission spectra in air (black, right axes) and in air in $50 \mathrm{mM} \mathrm{Na}_{2} \mathrm{SO}_{3} \mathrm{PBS}$ (blue, right axes) of (a) A-UL, (b) ApT-UL, (c) T-UL, and (d) pTA-UL with $10 \mathrm{~mW} 630 \mathrm{~nm}\left(80 \mathrm{~mW} \mathrm{~cm}^{-2}\right)$ at $20{ }^{\circ} \mathrm{C}$.

(before washing) showed that the resulting coated liposomes were monodisperse with an increase in hydrodynamic size of 17 $\mathrm{nm}$ with respect to the purified A-UL (Table 1), indicating that an additional layer of silica was deposited on A-UL. The DLS values did not change significantly for at least 1 week after preparation. TEM images showed monodispersed particles with an average particle diameter of $163 \mathrm{~nm}$, together with smaller clustered particles, which are probably silica nanoparticles (Figure 5). Similar to previous observations with A-UL, the particles dried out under intense electron irradiation in the vacuum of the TEM (Figure 5d), which allowed direct visualization of the solid silica shell around the liposome. Overall, method A successfully produced (organo)silica-coated liposomes that were monodispersed and stable in aqueous solution.
Preparation of Silica-Coated Liposomes-Method B. Method B involved the silica-coating of UL with TEOS under acidic catalytic conditions to make T-UL via a modified literature procedure. $^{28,29}$ Acid catalysis may result in more extensive condensation of the silica network, ${ }^{45}$ and thus improve oxygen protection. Clear solutions were obtained that did not aggregate visibly within 1 week, but an accurate size distribution by DLS could not be measured (PDI $=1.00$ ). The zeta potential of T-UL was found to be negative $(-33.4$ $\mathrm{mV}$ ). TEM imaging showed that T-UL consists of monodispersed particles with a rather broad size distribution (Figure S2). Much smaller particles $(<10 \mathrm{~nm})$ were also present, which are probably silica nanoparticles originating from TEOS condensation in solution instead of on the liposome surface. Interestingly, compared to A-UL and ApT-UL these particles show only little drying out under intense electron irradiation in 
a
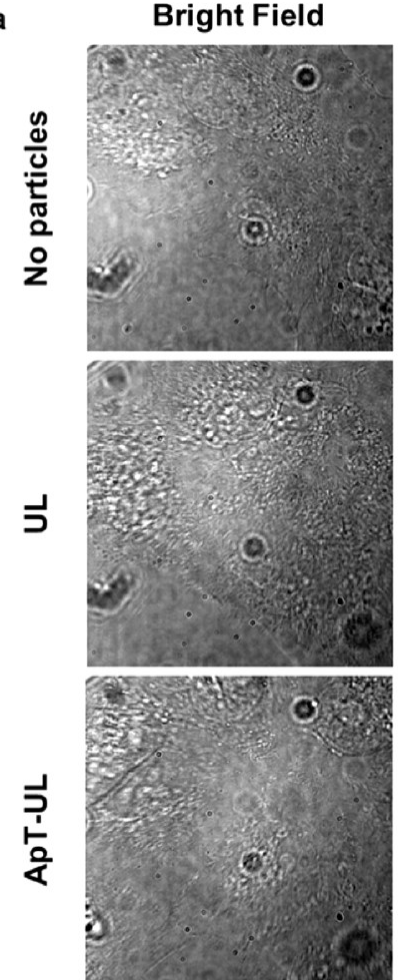

b

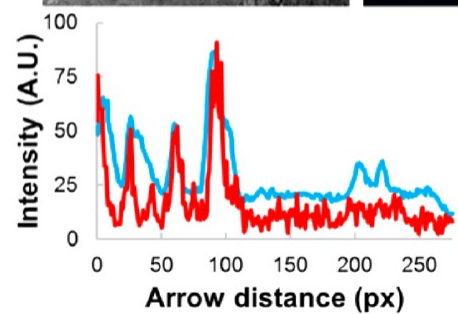

$\Lambda_{\text {exc }}=405 \mathrm{~nm}$
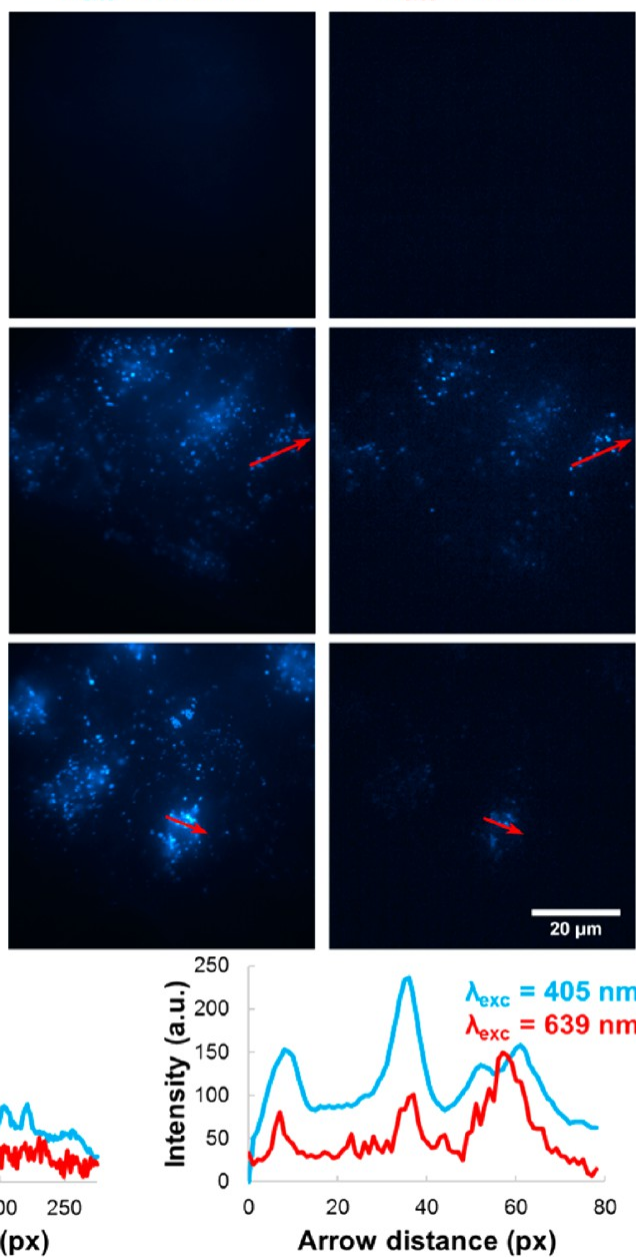

Figure 7. (a) Microscopy imaging in bright field mode at $100 \times$ magnification and with 405 or $639 \mathrm{~nm}\left(26 \mathrm{~W} \mathrm{~cm}^{-2}\right)$ excitation of living A549 cells treated with either medium only ("no particles"), UL, or ApT-UL at $1 \% \mathrm{O}_{2}, 7 \% \mathrm{CO}_{2}$, and $37^{\circ} \mathrm{C}$. (b) Intensity profiles of luminescence observed with 405 and $639 \mathrm{~nm}$ along the red arrows given in a, for samples UL (left) and ApT-UL (right).

the TEM. This may indicate that the silica network in these particles is indeed more condensed than in A-UL and ApT-UL. Overall, method B successfully produced silica-coated liposomes, albeit with a poorly defined particle diameter and poor particle purity.

Preparation of Silica-Coated Liposomes-Method C. Method C reversed the two synthesis steps of Method A: UL was first coated with prehydrolyzed TEOS to make pT-UL, and then additionally coated with APTES to make pTA-UL. The zeta-potential of $\mathbf{p T}$-UL and pTA-UL had similar negative values $(-18.8$ and $-17.0 \mathrm{mV}$, respectively). Aqueous samples containing pTA-UL were not very stable over time; aggregated particles were observed after a few days at room temperature. Freshly prepared pTA-UL had an average hydrodynamic size of $167 \mathrm{~nm}$. TEM imaging showed polygonal particles similar to ApT-UL, with an average particle diameter of $137 \mathrm{~nm}$ (Figure S3). Thus, although singly dispersed silica-coated liposomes were produced with method $\mathrm{C}$, the particles were of lower quality than ApT-UL from method A in terms of aggregation over time.

Spectroscopic Properties of Silica-Coated Liposomes in Solution. To evaluate whether the silica-coated liposome solutions produced upconversion, samples A-UL, ApT-UL, TUL, and pTA-UL were investigated with UV-vis absorption and emission spectroscopy $\left(\lambda_{\text {exc }}=630 \mathrm{~nm}, 10 \mathrm{~mW}, 80 \mathrm{~mW}\right.$ $\mathrm{cm}^{-2}$ ), see Figure 6. All absorption spectra matched the absorption spectrum of UL (Figure 3), ${ }^{1}$ which means that the silica-coating did not affect the molecular dyes. Emission spectra were first taken in air, after which sodium sulfite $\left(\mathrm{Na}_{2} \mathrm{SO}_{3}, 50 \mathrm{mM}\right)$ was added to scavenge ground-state oxygen and the emission spectra were recorded again. Without sulfite, for all solutions only very weak phosphorescence of PdTPTBP $\left(\lambda_{\mathrm{em}}=800 \mathrm{~nm}\right)$ was observed in comparison with $\mathrm{UL}$ in the presence of sulfite (Figure 3). However, upon addition of sulfite, all samples directly exhibited much more intense phosphorescence of PdTPTBP and intense upconverted emission of perylene at $474 \mathrm{~nm}$. In a second experiment, to ascertain that the silica-coating had not destroyed the lipid bilayer, the temperature dependence of phosphorescence and upconversion was recorded between 10 and $35{ }^{\circ} \mathrm{C}$ in the presence of sulfite. ${ }^{39,69}$ If the lipid bilayer would still be intact, a steep increase in upconversion and decrease of phosphorescence around the lipid bilayer main transition temperature $\left(T_{\mathrm{m}} \approx 25^{\circ} \mathrm{C}\right)$ would be expected, just as was observed for UL (Figure 3). Indeed, in all cases, the thermo-photophysical behavior was similar to UL, confirming that the lipid bilayer was still intact (Figure S4). Overall, despite the lipid bilayer being intact inside the particles, it is clear that none of the 


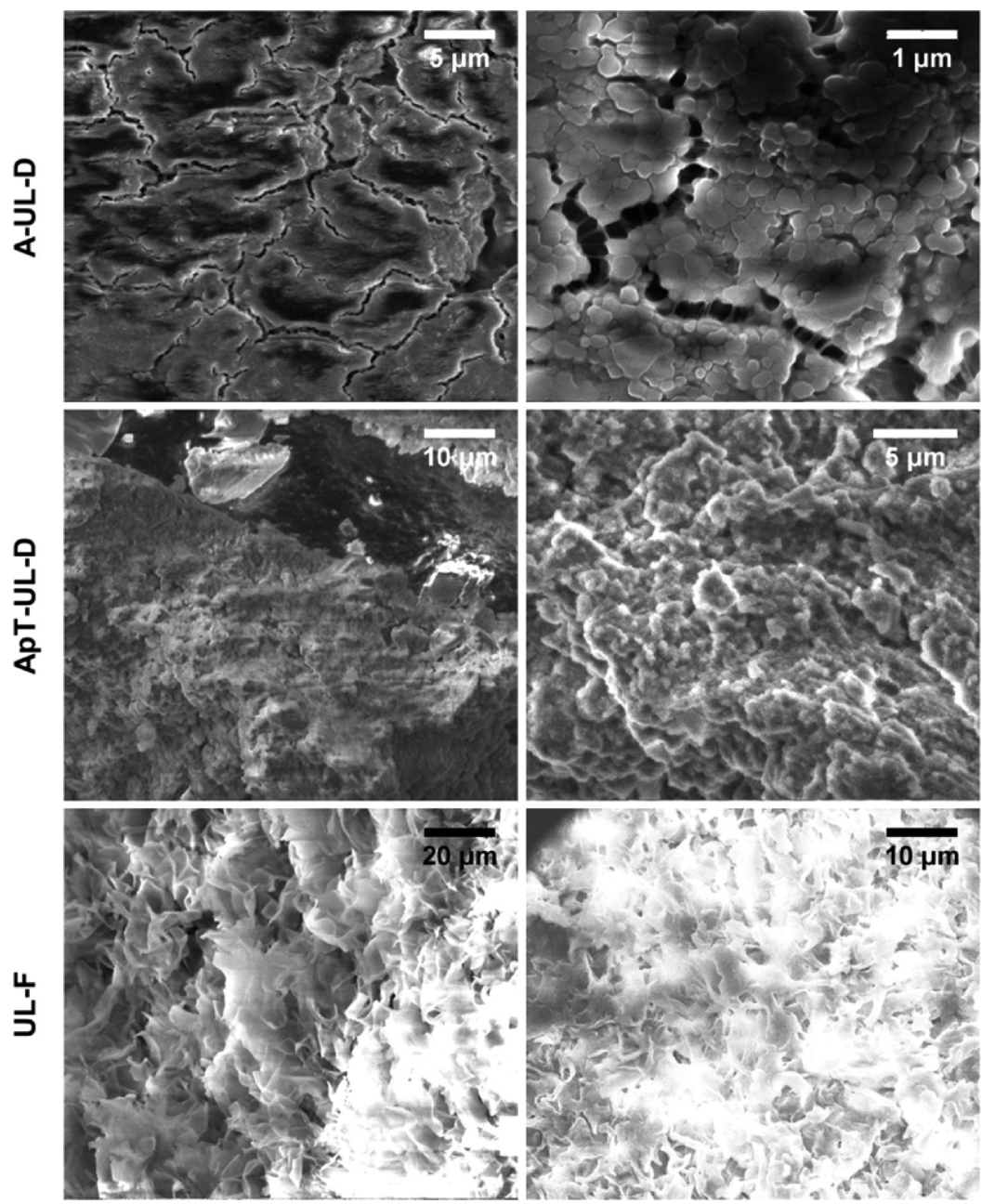

Figure 8. SEM micrographs of dried (organo)silica-coated liposomes A-UL-D and ApT-UL-D, and freeze-dried liposomes UL-F.

(organo)silica layers around the liposomes were capable of blocking oxygen. This must mean that the organo(silica) coating is either porous to oxygen or incomplete, because the capability of upconversion, which takes place inside the particles, is affected by the sodium sulfite added to the solution outside the particles. This result can only be explained if oxygen is able to diffuse freely across the organosilica-coating or across the patches that have remained uncoated.

Other silica-coated hybrid systems for TTA-UC have been reported. For example, Liu et al. described acid-catalyzed silicacoating of TTA-UC dye-loaded Pluronic F-127 micelles with TEOS (similar to method B). ${ }^{28,29}$ They showed that the watersoluble particles performed upconversion, but did not mention oxygen quenching of the process at all. In fact, Wang and coworkers used identical particles that were functionalized with two dyes for ratiometric oxygen sensing in cells. ${ }^{70}$ Obviously, such particles must be oxygen permeable if they are used for oxygen sensing. Kwon et al. discussed the oxygen sensitivity of an upconverting oleic acid-core silica-shell nanocapsules, with a silica shell thickness of $12-23 \mathrm{~nm}^{23}$ Although the system was capable of upconversion in air, it was not the relatively thick silica shell that protected the dyes from oxygen, but the oleic acid that is able to scavenge oxygen; without oleic acid, no upconversion was observed. Thus, so far, in all TTA-UC systems with nanometer-thick silica shells, silica offers no protection from oxygen. Our results seem to be yet another example that nanosize silica layers, made by various methods, is not able to block the diffusion of molecular oxygen in aqueous solution.

Upconversion with Silica-Coated Liposomes in Cells. Although TTA-UC in liposomes or (organo)silica-coated liposomes in solution is too oxygen-sensitive, it would be incorrect to assume that they do not function in biological systems. Indeed, TTA-UC has been shown before to occur in vitro and in vivo with nanoparticle systems that are oxygen sensitive as well. ${ }^{71,72} \mathrm{Up}$ to now, a reasonable explanation has not yet been provided in the literature why such particles are able to produce upconversion in biological systems. Possibly, TTA-UC is facilitated by the presence of endogenous antioxidants that are able to lower the local oxygen concentration by consuming ground state oxygen or singlet oxygen. Furthermore, the silica shell may actually offer protection from oxygen in a biological situation in which oxygen is present at a lower concentration than in an aqueous dispersion. With this in mind, A549 lung carcinoma cells were treated with UL, A-UL, or ApT-UL and then imaged with (upconversion) luminescence microscopy. For these experiments, perylene was substituted by 2,5,8,11-tetra(tert-butyl)perylene (TBP, Figure 1) to prevent the annihilator from escaping the liposomes, which is known to occur for perylene. ${ }^{73}$ First of all, uptake of the particles was investigated after $24 \mathrm{~h}$ incubation by regular fluorescence microscopy (20X magnifi- 
cation) to image the emission of TBP $\left(\lambda_{\text {exc }}=377 \mathrm{~nm}\right)$, see Figure S5. For both liposomes and silica-coated liposomes, the appearance of blue fluorescence throughout the cytosol confirmed the cellular uptake of the particles. The differences in zeta-potential and the presence of the (organo)silica-coating did not seem to affect the particle uptake significantly. Furthermore, upon systematic inspection of the treated cells with light microscopy at $4 \times$ magnification, no significant amount of dead or damaged cells were observed, thereby raising no concerns about particle toxicity. In a second experiment, the cells were incubated with either UL or ApTUL for $24 \mathrm{~h}$ and then imaged at $100 \times$ magnification with 405 and $639 \mathrm{~nm}\left(26 \mathrm{~W} \mathrm{~cm}^{-2}\right)$ excitation under poorly oxygenated conditions $\left(1 \% \mathrm{O}_{2}\right)$, see Figure 7 . For both UL and ApT-UL, under $405 \mathrm{~nm}$ excitation, bright fluorescent spots were observed, marking the locations of the TBP dye. Given that the usual uptake mechanism of liposomes is endocytosis, we assign these spots to be endo- and lysosomes. Interestingly, upon $639 \mathrm{~nm}$ excitation and observing between 450 to $575 \mathrm{~nm}$, upconverted emission was observed at the same locations as that observed for TBP fluorescence upon $405 \mathrm{~nm}$ excitation (Figure 7). Comparable upconversion intensities were observed in vitro for both UL and ApT-UL, which required excitation intensities at $26 \mathrm{~W} \mathrm{~cm}^{-2}$ to be observable. No upconversion could be observed below this excitation intensity (Figure S6). It was noticed that at $26 \mathrm{~W} \mathrm{~cm}^{-2}$, the upconversion intensity varied significantly from cell to cell, which possibly reflects differences in oxygenation levels and concentration of endogenous antioxidants. Overall, UL and ApT-UL performed upconversion in A549 cells, but silica coating of the liposomes improved neither uptake nor upconversion luminescence intensity in vitro.

Dried Upconverting Silica-Coated Liposomes. One of the reasons why the silica-coating does not act as an oxygen barrier may be the coating thickness. How thick should the silica-coating be to become a barrier for oxygen? Attempts were undertaken to grow extra layers of silica on ApT-UL, but this led inevitably to aggregation of the particles in solution. As an alternative, it was decided to prepare a solid silica-liposome composite material by drying unpurified A-UL and ApT-UL samples, i.e. without removing the excess of APTES or preTEOS, in an oven at $50{ }^{\circ} \mathrm{C}$ overnight. The new samples were called A-UL-D and ApT-UL-D, respectively. As silica-free control, liposomes UL were freeze-dried (sample UL-F). For A-UL-D and ApT-UL-D, heat-drying drives the condensation equilibrium of the (organo)silica network to the fully condensed species by the removal of $\mathrm{H}_{2} \mathrm{O}$ and $\mathrm{EtOH}$, making a dense silica material. Interestingly, A-UL-D could be completely redispersed in PBS using sonication for $25 \mathrm{~min}$, resulting in a clear solution with $137 \mathrm{~nm}$ diameter particles, while the same treatment for ApT-UL-D resulted in only partial redispersion with visible large aggregates (Figure S7). After removal of these aggregates with low-speed centrifugation, the solution contained particles with an average hydrodynamic diameter of $316 \mathrm{~nm}$. This is evidence that in A-UL-D, the individual organosilica-coated liposomes are not covalently attached to each other but are embedded in a loose matrix that is easily disrupted, whereas in ApT-UL-D the individual particles are tightly bound together by silica.

The solids were characterized with scanning electron microscopy (SEM) and ${ }^{29} \mathrm{Si}$ NMR. Figure 8 shows SEM images of the solids. A-UL-D consisted of a mesh of sphericalpolygonal particles within the same size range as the water dispersed A-UL particles observed by TEM. The ${ }^{29}$ Si NMR spectrum (Figure S8) shows a broad peak at $-68 \mathrm{ppm}$, corresponding to the fully condensed $\left(\mathrm{T}_{3}\right)$ organosilica product of APTES. ${ }^{67,94}$ In comparison, ApT-UL-D had a more coarse structure, and individual particles could not be distinguished. This is consistent with the hypothesis that ApT-UL particles are embedded in a matrix of amorphous silica. The ${ }^{29} \mathrm{Si} \mathrm{NMR}$ spectrum of this material showed two peaks at -106 and -96 $\mathrm{ppm}$, corresponding to the triple-condensed $\left(\mathrm{Q}_{3}\right)$ and doublecondensed $\left(\mathrm{Q}_{2}\right)$ silica products of TEOS (Figure S8). Thus, the silica matrix of ApT-UL-D is not fully condensed. No signals originating from condensed APTES were detected, which emphasizes that the silica vs organosilica ratio is very high. In contrast, SEM images of UL-F suggested that this sticky solid consisted of a network of broken lipid bilayers, which underlines that the silica shell around the liposomes is necessary to conserve the nanostructure of the silica-coated liposomes inside the dried composite materials.

To investigate the integrity of the liposomes inside the material, we performed thermogravimetric analysis (TGA) on all solid samples. If the liposomes would be intact, i.e. defined as a lipid bilayer surrounding an aqueous interior, it was expected that water would escape from $\sim 100{ }^{\circ} \mathrm{C}$ onward, followed by thermal decomposition of the phospholipids at a higher temperature. Figure 9 shows the TGA curves of freeze-

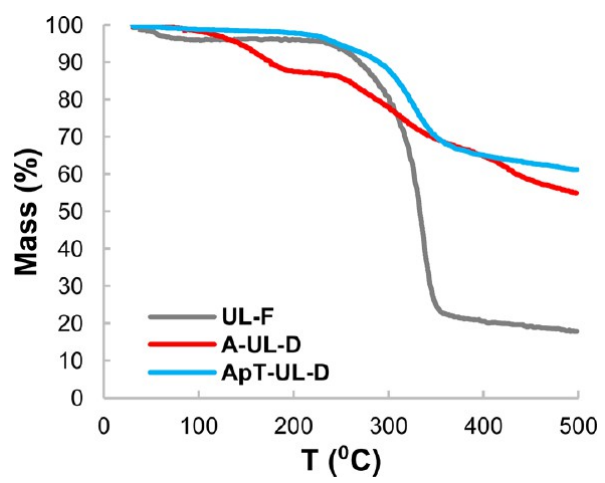

Figure 9. Thermogravimetric analysis plots from 30 to $500{ }^{\circ} \mathrm{C}\left(10^{\circ} \mathrm{C}\right.$ $\mathrm{min}^{-1}$ in air) of freeze-dried liposomes UL-F, and heat-dried (organo)silica-coated liposomes A-UL-D and ApT-UL-D.

dried liposomes UL-F, and heat-dried (organo)silica-coated liposomes A-UL-D and ApT-UL-D. The mass of UL-F reduces by $75 \%$ between 230 and $350{ }^{\circ} \mathrm{C}$, indicating the expected thermal decomposition of the phospholipids. The curve for ApT-UL-D is very similar to UL-F, but with a $40 \%$ mass reduction between 230 and $350{ }^{\circ} \mathrm{C}$. The higher residual mass is attributed to the empty residual silica shells, which evidently do not decompose at this temperature. No mass decrease was observed between 30 and $230{ }^{\circ} \mathrm{C}$, indicating the absence of water and thus the absence of intact liposomes inside this material. The TGA curve for A-UL-D shows a gradual mass reduction of $13 \%$ between 100 and $200{ }^{\circ} \mathrm{C}$, and again a second mass reduction from $230{ }^{\circ} \mathrm{C}$ onward. The mass reductions between 100 and $200{ }^{\circ} \mathrm{C}$ suggest the loss of water. However, the theoretical percentage of water mass, assuming $100 \%$ synthesis yield and $130 \mathrm{~nm}$ diameter liposomes, would amount to $50-60 \%$. Therefore, these data indicate that only a relatively small part of A-UL-D consist of intact liposomes and that the (organo)silica matrix around the liposomes was not able to prevent drying out of the aqueous interior of the liposomes. 

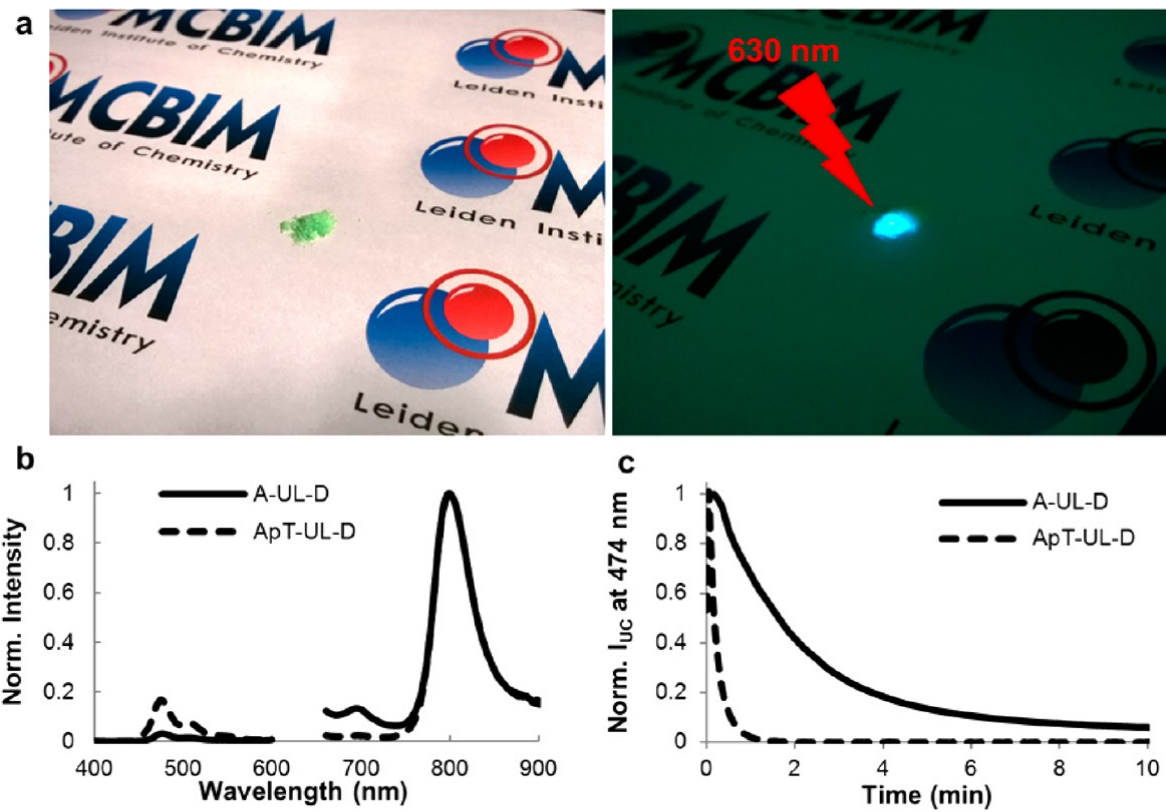

d

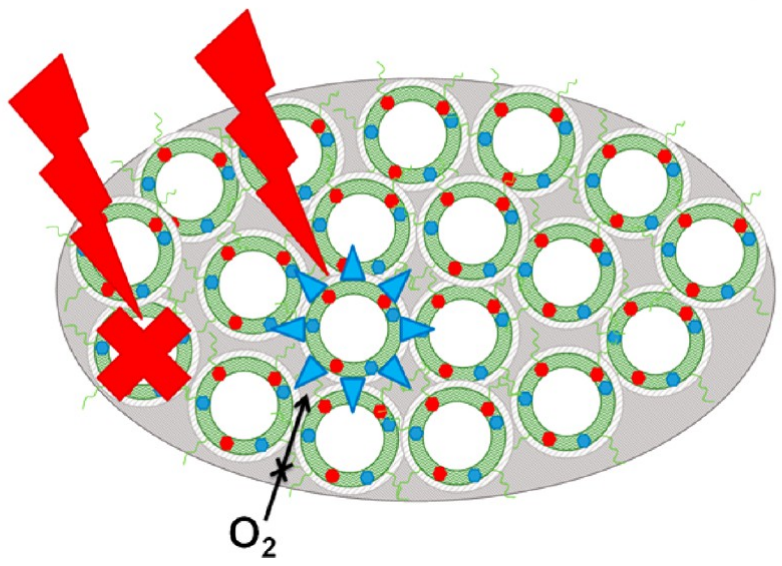

Figure 10. Upconversion with heat-dried silica-coated liposomes. (a) Photographs of heat-dried organosilica-coated liposomes A-UL-D in ambient light (left) and irradiated with red light and photographed with a $575 \mathrm{~nm}$ short pass filter in front of the camera (right). (b) Emission spectra of AUL-D (solid) and ApT-UL-D (dashed) under $630 \mathrm{~nm}$ irradiation, normalized at $800 \mathrm{~nm}$. (c) Typical time traces of the normalized upconversion intensity $\left(I_{\mathrm{UC}}\right)$ at $474 \mathrm{~nm}$ of A-UL-D (solid) and ApT-UL-D (dashed) under continuous $630 \mathrm{~nm}$ irradiation. All experiments were performed at 20 ${ }^{\circ} \mathrm{C}$ with $30 \mathrm{~mW} 630 \mathrm{~nm}$ irradiation $\left(0.66 \mathrm{~W} \mathrm{~cm}^{-2}\right)$. (d) Schematic representation of the upconverting solids in which silica-coated liposomes are embedded in a solid matrix. It is proposed that upconversion only occurs in the most deeply buried liposomes, which are the best protected from oxygen.

Regardless of the fact that the water inside the material is lost, the residual fragments of dye-doped lipid bilayer inside the solid may still be able to perform upconversion. As an initial test, A-UL-D powder was irradiated with $630 \mathrm{~nm}$ in air, and surprisingly, blue luminescence was clearly visible after blocking the excitation light (Figure 10a). Emission spectroscopy $\left(\lambda_{\text {exc }}=\right.$ $630 \mathrm{~nm}, 30 \mathrm{~mW}, 0.66 \mathrm{~W} \mathrm{~cm}^{-2}$ ) confirmed that A-UL-D and ApT-UL-D were indeed producing upconverted luminescence under red light irradiation (Figure 10b). In contrast, no upconversion in air was detected for freeze-dried liposomes UL-F (Figure S9). The upconversion emission in A-UL-D and ApT-UL-D was not very durable; bleaching occurred within minutes at $0.66 \mathrm{~W} \mathrm{~cm}^{-2}$ irradiance (Figure 10c). Time-traces of the upconversion intensity revealed that the emission was more long-lasting for A-UL-D, whereas all upconversion luminescence had bleached within 2 min for ApT-UL-D, $40 \%$ of the start intensity still remained for A-UL-D. This difference may be caused by the greater amount of primary amine groups in AUL-D, which are known to chemically quench singlet oxygen.
Such a chemical reaction consumes dioxygen and therefore may contribute to an oxygen-low environment inside the material upon irradiation. ${ }^{75,76}$ Nonetheless, the relative instability of the upconversion emission underlines that even in such bulk materials, (organo)silica does not completely obstruct the diffusion of oxygen. As a final experiment, redispersed A-UL-D and ApT-UL-D were investigated with UV-vis absorption and emission spectroscopy (Figure S10). Just as for A-UL and ApTUL, upconversion was only visible after removal of oxygen by adding sodium sulfite. Assuming that redispersion did not affect the integrity of the silica coating, these results suggest that even heat-dried (organo)silica is porous to $\mathrm{O}_{2}$ when resolubilized in aqueous solution, and it is neither the higher degree of condensation nor the increased silica thickness that is responsible for the oxygen protection in the parent solids. Instead, it is proposed that the upconversion in the solids originates from the most deeply buried particles, which are protected from air by surrounding particles that themselves are not generating upconversion (Figure 10d). It is thus clear that a 
much thicker or compact layer of (organo)silica is necessary to protect silica-coated liposomes in solution and in solids. Finally, it must be emphasized that these results are rather preliminary and that the preparation method for obtaining these silicacoated materials can be greatly improved. We foresee that an optimized drying procedure would yield solids with higher degree of silica condensation and in which all the water inside the liposomes remains trapped. Then, the addition of watersoluble antioxidants to the aqueous interior of the liposomes before silicification and drying, which would end up inside the material, would greatly enhance the upconversion quantum yield and stability in air. ${ }^{41,63}$ Such highly tunable nanocomposite materials, consisting of silica, phospholipids, and water, would effectively allow air-sensitive photophysical processes to take place in a solid state material.

\section{CONCLUSIONS}

Monodisperse (organo)silica-coated liposomes were prepared that can be used to upconvert red light to blue light by means of triplet-triplet annihilation upconversion. The silica-coating did not prevent the upconversion to be quenched by molecular oxygen in solution. Furthermore, the upconverted blue light could be imaged in living A549 cells in hypoxic conditions without causing cytotoxicity, but the luminescence was not more intense than in control cells that had been treated with uncoated upconverting liposomes. However, when the (organo)silica-coated liposomes were heat-dried in the presence of excess (organo)silica precursor, solids were obtained that could perform upconversion in air. Our results suggest that the (organo)silica shell of the silica-coated liposomes in solution needs to be much thicker and/or compact to protect the upconversion from oxygen in a biological setting. This work represents exciting examples of the combination of phospholipids, water, and silica for the construction of tunable upconverting nanoparticles and materials. Such hybrid systems combine the favorable properties of their constituents, and may eventually be used in applications such as drug delivery, cell imaging, photocatalysis, and solar energy harvesting.

\section{ASSOCIATED CONTENT}

\section{S Supporting Information}

The Supporting Information is available free of charge on the ACS Publications website at DOI: 10.1021/acsbiomaterials.6b00678.

Figures S1-S12 (PDF)

\section{AUTHOR INFORMATION}

\section{Corresponding Author}

*E-mail: bonnet@chem.leidenuniv.nl.

\section{ORCID}

Sylvestre Bonnet: 0000-0002-5810-3657

\section{Notes}

The authors declare no competing financial interest.

\section{ACKNOWLEDGMENTS}

This work was supported by the Dutch Organization for Scientific Research (NWO-CW) via a VIDI grant to S.B. The European Research Council is kindly acknowledged for a Starting Grant to S.B. Prof. E. Bouwman is gratefully acknowledged for her support and input. The COST action
CM1105 "Functional metal complexes that bind to biomolecules" is gratefully acknowledged for stimulating scientific discussion.

\section{REFERENCES}

(1) Askes, S. H. C.; Bahreman, A.; Bonnet, S. Activation of a Photodissociative Ruthenium Complex by Triplet-Triplet Annihilation Upconversion in Liposomes. Angew. Chem., Int. Ed. 2014, 53 (4), 1029-1033.

(2) Askes, S. H. C.; Kloz, M.; Bruylants, G.; Kennis, J. T.; Bonnet, S. Triplet-triplet annihilation upconversion followed by FRET for the red light activation of a photodissociative ruthenium complex in liposomes. Phys. Chem. Chem. Phys. 2015, 17 (41), 27380-90.

(3) Chen, Z.; Sun, W.; Butt, H.-J.; Wu, S. UpconvertingNanoparticle-Assisted Photochemistry Induced by Low-Intensity Near-Infrared Light: How Low Can We Go? Chem. - Eur. J. 2015, 21 (25), 9165-9170.

(4) Zhou, J.; Liu, Q.; Feng, W.; Sun, Y.; Li, F. Upconversion Luminescent Materials: Advances and Applications. Chem. Rev. 2014, 115 (1), 395-465.

(5) Xia, L.; Kong, X.; Liu, X.; Tu, L.; Zhang, Y.; Chang, Y.; Liu, K.; Shen, D.; Zhao, H.; Zhang, H. An upconversion nanoparticle - Zinc phthalocyanine based nanophotosensitizer for photodynamic therapy. Biomaterials 2014, 35 (13), 4146-4156.

(6) Ruggiero, E.; Hernandez-Gil, J.; Mareque-Rivas, J. C.; Salassa, L. Near infrared activation of an anticancer PtIV complex by Tm-doped upconversion nanoparticles. Chem. Commun. 2015, 51, 2091.

(7) Mahato, P.; Yanai, N.; Sindoro, M.; Granick, S.; Kimizuka, N. Preorganized Chromophores Facilitate Triplet Energy Migration, Annihilation and Upconverted Singlet Energy Collection. J. Am. Chem. Soc. 2016, 138, 6541.

(8) Liu, Q.; Yin, B.; Yang, T.; Yang, Y.; Shen, Z.; Yao, P.; Li, F. A General Strategy for Biocompatible, High-Effective Upconversion Nanocapsules Based on Triplet-Triplet Annihilation. J. Am. Chem. Soc. 2013, 135 (13), 5029-5037.

(9) Kwon, O. S.; Song, H. S.; Conde, J.; Kim, H.-i.; Artzi, N.; Kim, J.H. Dual-Color Emissive Upconversion Nanocapsules for Differential Cancer Bioimaging In Vivo. ACS Nano 2016, 10 (1), 1512-21.

(10) Singh-Rachford, T. N.; Castellano, F. N. Photon upconversion based on sensitized triplet-triplet annihilation. Coord. Chem. Rev. 2010, 254 (21-22), 2560-2573.

(11) Schmidt, T. W.; Castellano, F. N. Photochemical Upconversion: The Primacy of Kinetics. J. Phys. Chem. Lett. 2014, 5 (22), 4062-4072.

(12) Monguzzi, A.; Tubino, R.; Hoseinkhani, S.; Campione, M.; Meinardi, F. Low power, non-coherent sensitized photon upconversion: modelling and perspectives. Phys. Chem. Chem. Phys. 2012, 14 (13), 4322-4332.

(13) Yanai, N.; Kimizuka, N. Recent emergence of photon upconversion based on triplet energy migration in molecular assemblies. Chem. Commun. 2016, 52 (31), 5354-5370.

(14) Hisamitsu, S.; Yanai, N.; Kimizuka, N. Photon-Upconverting Ionic Liquids: Effective Triplet Energy Migration in Contiguous Ionic Chromophore Arrays. Angew. Chem., Int. Ed. 2015, 54 (39), 11550-4.

(15) Mahato, P.; Monguzzi, A.; Yanai, N.; Yamada, T.; Kimizuka, N. Fast and long-range triplet exciton diffusion in metal-organic frameworks for photon upconversion at ultralow excitation power. Nat. Mater. 2015, 14 (9), 924-930.

(16) Lee, S. H.; Thévenaz, D. C.; Weder, C.; Simon, Y. C. Glassy poly(methacrylate) terpolymers with covalently attached emitters and sensitizers for low-power light upconversion. J. Polym. Sci., Part A: Polym. Chem. 2015, 53 (14), 1629-1639.

(17) Duan, P.; Yanai, N.; Nagatomi, H.; Kimizuka, N. Photon Upconversion in Supramolecular Gel Matrices: Spontaneous Accumulation of Light-Harvesting Donor-Acceptor Arrays in Nanofibers and Acquired Air Stability. J. Am. Chem. Soc. 2015, 137 (5), 1887-94.

(18) Duan, P.; Yanai, N.; Kimizuka, N. Photon Upconverting Liquids: Matrix-Free Molecular Upconversion Systems Functioning in Air. J. Am. Chem. Soc. 2013, 135 (51), 19056-19059. 
(19) Svagan, A. J.; Busko, D.; Avlasevich, Y.; Glasser, G.; Baluschev, S.; Landfester, K. Photon energy upconverting nanopaper: a bioinspired oxygen protection strategy. ACS Nano 2014, 8 (8), 8198-207.

(20) Kim, J.-H.; Kim, J.-H. Triple-Emulsion Microcapsules for Highly Efficient Multispectral Upconversion in the Aqueous Phase. ACS Photonics 2015, 2 (5), 633-638.

(21) Huang, Z.; Li, X.; Mahboub, M.; Hanson, K.; Nichols, V.; Le, H.; Tang, M. L.; Bardeen, C. J. Hybrid molecule-nanocrystal photon upconversion across the visible and near-infrared. Nano Lett. 2015, 15 (8), 5552-7.

(22) Majek, M.; Faltermeier, U.; Dick, B.; Pérez-Ruiz, R.; Jacobi von Wangelin, A. Application of Visible-to-UV Photon Upconversion to Photoredox Catalysis: The Activation of Aryl Bromides. Chem. - Eur. J. 2015, 21 (44), 15496-15501.

(23) Kwon, O. S.; Kim, J. H.; Cho, J. K.; Kim, J. H. Triplet-triplet annihilation upconversion in CdS-decorated $\mathrm{SiO} 2$ nanocapsules for sub-bandgap photocatalysis. ACS Appl. Mater. Interfaces 2015, 7 (1), $318-25$.

(24) Monguzzi, A.; Borisov, S. M.; Pedrini, J.; Klimant, I.; Salvalaggio, M.; Biagini, P.; Melchiorre, F.; Lelii, C.; Meinardi, F. Efficient Broadband Triplet-Triplet Annihilation-Assisted Photon Upconversion at Subsolar Irradiance in Fully Organic Systems. Adv. Funct. Mater. 2015, 25 (35), 5617-5624.

(25) Simpson, C.; Clarke, T.; MacQueen, R. W.; Cheng, Y. Y.; Trevitt, A.; Mozer, A. J.; Wagner, P.; Schmidt, T. W.; Nattestad, A. An Intermediate Band Dye-sensitised Solar Cell Using Triplet-Triplet Annihilation. Phys. Chem. Chem. Phys. 2015, 17, 24826.

(26) Hill, S. P.; Banerjee, T.; Dilbeck, T.; Hanson, K. Photon Upconversion and Photocurrent Generation via Self-Assembly at Organic-Inorganic Interfaces. J. Phys. Chem. Lett. 2015, 6 (22), 45104517.

(27) Nattestad, A.; Cheng, Y. Y.; MacQueen, R. W.; Schulze, T. F.; Thompson, F. W.; Mozer, A. J.; Fückel, B.; Khoury, T.; Crossley, M. J.; Lips, K.; Wallace, G. G.; Schmidt, T. W. Dye-Sensitized Solar Cell with Integrated Triplet-Triplet Annihilation Upconversion System. J. Phys. Chem. Lett. 2013, 4 (12), 2073-2078.

(28) Liu, Q.; Feng, W.; Yang, T.; Yi, T.; Li, F. Upconversion luminescence imaging of cells and small animals. Nat. Protoc. 2013, 8 (10), 2033-2044.

(29) Liu, Q.; Yang, T.; Feng, W.; Li, F. Blue-Emissive Upconversion Nanoparticles for Low-Power-Excited Bioimaging in Vivo. J. Am. Chem. Soc. 2012, 134 (11), 5390-5397.

(30) Nagai, A.; Miller, J. B.; Kos, P.; Elkassih, S.; Xiong, H.; Siegwart, D. J. Tumor Imaging Based on Photon Upconversion of $\mathrm{Pt}(\mathrm{II})$ Porphyrin Rhodamine Co-modified NIR Excitable Cellulose Enhanced by Aggregation. ACS Biomater. Sci. Eng. 2015, 1 (12), 1206-1210.

(31) Wohnhaas, C.; Mailänder, V.; Dröge, M.; Filatov, M. A.; Busko, D.; Avlasevich, Y.; Baluschev, S.; Miteva, T.; Landfester, K.; Turshatov, A. Triplet-Triplet Annihilation Upconversion Based Nanocapsules for Bioimaging Under Excitation by Red and Deep-Red Light. Macromol. Biosci. 2013, 13 (10), 1422-1430.

(32) Wohnhaas, C.; Turshatov, A.; Mailänder, V.; Lorenz, S.; Baluschev, S.; Miteva, T.; Landfester, K. Annihilation Upconversion in Cells by Embedding the Dye System in Polymeric Nanocapsules. Macromol. Biosci. 2011, 11 (6), 772-778.

(33) Sawant, R. R.; Torchilin, V. P. Liposomes as 'smart' pharmaceutical nanocarriers. Soft Matter 2010, 6 (17), 4026-4044.

(34) Derycke, A. S. L.; de Witte, P. A. M. Liposomes for photodynamic therapy. Adv. Drug Delivery Rev. 2004, 56 (1), 17-30.

(35) Allen, T. M.; Cullis, P. R. Liposomal drug delivery systems: from concept to clinical applications. Adv. Drug Delivery Rev. 2013, 65 (1), $36-48$.

(36) Feldmann, H. J.; Molls, M.; Vaupel, P. Blood flow and oxygenation status of human tumors. Strahlenther. Onkol. 1999, 175 (1), 1-9.

(37) Vaupel, P.; Kallinowski, F.; Okunieff, P. Blood Flow, Oxygen and Nutrient Supply, and Metabolic Microenvironment of Human Tumors: A Review. Cancer Res. 1989, 49 (23), 6449-6465.
(38) Graves, E. E.; Vilalta, M.; Cecic, I. K.; Erler, J. T.; Tran, P. T.; Felsher, D.; Sayles, L.; Sweet-Cordero, A.; Le, Q.-T.; Giaccia, A. J. Hypoxia in Models of Lung Cancer: Implications for Targeted Therapeutics. Clin. Cancer Res. 2010, 16 (19), 4843-4852.

(39) Penconi, M.; Gentili, P. L.; Massaro, G.; Elisei, F.; Ortica, F. A triplet-triplet annihilation based up-conversion process investigated in homogeneous solutions and oil-in-water microemulsions of surfactant. Photochem. Photobiol. Sci. 2014, 13, 48-61.

(40) Kim, J.-H.; Deng, F.; Castellano, F. N.; Kim, J.-H. Red-to-Blue/ Cyan/Green Upconverting Microcapsules for Aqueous- and DryPhase Color Tuning and Magnetic Sorting. ACS Photonics 2014, 1 (4), 382-388.

(41) Mongin, C.; Golden, J. H.; Castellano, F. N. Liquid PEG Polymers Containing Antioxidants: A Versatile Platform for Studying Oxygen-Sensitive Photochemical Processes. ACS Appl. Mater. Interfaces 2016, 8, 24038.

(42) Wohnhaas, C.; Friedemann, K.; Busko, D.; Landfester, K.; Baluschev, S.; Crespy, D.; Turshatov, A. All Organic Nanofibers As Ultralight Versatile Support for Triplet-Triplet Annihilation Upconversion. ACS Macro Lett. 2013, 2 (5), 446-450.

(43) Marsico, F.; Turshatov, A.; Peköz, R.; Avlasevich, Y.; Wagner, M.; Weber, K.; Donadio, D.; Landfester, K.; Baluschev, S.; Wurm, F. R. Hyperbranched Unsaturated Polyphosphates as Protective Matrix for Long-Term Photon Upconversion in Air. J. Am. Chem. Soc. 2014, 136, 11057.

(44) Brinker, C. J.; Scherer, G. W. Sol-Gel Science: The Physics and Chemistry of Sol-Gel Processing; Academic Press: Cambridge, MA, 1990.

(45) Brinker, C. J. Hydrolysis and Condensation of Silicates - Effects on Structure. J. Non-Cryst. Solids 1988, 100 (1-3), 31-50.

(46) Barbe, C.; Bartlett, J.; Kong, L. G.; Finnie, K.; Lin, H. Q.; Larkin, M.; Calleja, S.; Bush, A.; Calleja, G. Silica particles: A novel drugdelivery system. Adv. Mater. 2004, 16 (21), 1959-1966.

(47) Lucky, S. S.; Soo, K. S.; Zhang, Y. Nanoparticles in Photodynamic Therapy. Chem. Rev. 2015, 115, 1990.

(48) Frasconi, M.; Liu, Z. C.; Lei, J. Y.; Wu, Y. L.; Strekalova, E.; Malin, D.; Ambrogio, M. W.; Chen, X. Q.; Botros, Y. Y.; Cryns, V. L.; Sauvage, J. P.; Stoddart, J. F. Photoexpulsion of Surface-Grafted Ruthenium Complexes and Subsequent Release of Cytotoxic Cargos to Cancer Cells from Mesoporous Silica Nanoparticles. J. Am. Chem. Soc. 2013, 135 (31), 11603-11613.

(49) Leterrier, Y. Durability of nanosized oxygen-barrier coatings on polymers - Internal stresses. Prog. Mater. Sci. 2003, 48 (1), 1-55.

(50) Mirenda, M.; Levi, V.; Bossi, M. L.; Bruno, L.; Bordoni, A. V.; Regazzoni, A. E.; Wolosiuk, A. Temperature response of luminescent tris(bipyridine)ruthenium(II)-doped silica nanoparticles. J. Colloid Interface Sci. 2013, 392 (0), 96-101.

(51) Zhang, D.; Wu, Z.; Xu, J.; Liang, J.; Li, J.; Yang, W. Tuning the Emission Properties of $\mathrm{Ru}($ phen)32+ Doped Silica Nanoparticles by Changing the Addition Time of the Dye during the Stöber Process. Langmuir 2010, 26 (9), 6657-6662.

(52) Rossi, L. M.; Shi, L.; Quina, F. H.; Rosenzweig, Z. Stöber Synthesis of Monodispersed Luminescent Silica Nanoparticles for Bioanalytical Assays. Langmuir 2005, 21 (10), 4277-4280.

(53) Folliet, N.; Roiland, C.; Bégu, S.; Aubert, A.; Mineva, T.; Goursot, A.; Selvaraj, K.; Duma, L.; Tielens, F.; Mauri, F.; Laurent, G.; Bonhomme, C.; Gervais, C.; Babonneau, F.; Azaïs, T. Investigation of the Interface in Silica-Encapsulated Liposomes by Combining Solid State NMR and First Principles Calculations. J. Am. Chem. Soc. 2011, 133 (42), 16815-16827.

(54) Begu, S.; Durand, R.; Lerner, D. A.; Charnay, C.; TournePeteilh, C.; Devoisselle, J. M. Preparation and characterization of siliceous material using liposomes as template. Chem. Commun. 2003, No. 5, 640-641.

(55) Hubert, D. H. W.; Jung, M.; Frederik, P. M.; Bomans, P. H. H.; Meuldijk, J.; German, A. L. Vesicle-Directed Growth of Silica. Adv. Mater. 2000, 12 (17), 1286-1290.

(56) Beloglazova, N. V.; Goryacheva, O. A.; Speranskaya, E. S.; Aubert, T.; Shmelin, P. S.; Kurbangaleev, V. R.; Goryacheva, I. Y.; De 
Saeger, S. Silica-coated liposomes loaded with quantum dots as labels for multiplex fluorescent immunoassay. Talanta 2015, 134 (0), 120125.

(57) Beloglazova, N. V.; Goryacheva, I. Y.; Shmelin, P. S.; Kurbangaleev, V.; De Saeger, S. Preparation and characterization of stable phospholipid-silica nanostructures loaded with quantum dots. $J$. Mater. Chem. B 2015, 3 (2), 180-183.

(58) Corma, A.; Díaz, U.; Arrica, M.; Fernández, E.; Ortega, Í. Organic-Inorganic Nanospheres with Responsive Molecular Gates for Drug Storage and Release. Angew. Chem., Int. Ed. 2009, 48 (34), 6247-6250.

(59) Shen, S.; Yang, L.; Lu, Y.; Chen, J.-G.; Song, S.; Hu, D.; Parikh, A. A New Route to Liposil Formation by an Interfacial Sol-Gel Process Confined by Lipid Bilayer. ACS Appl. Mater. Interfaces 2015, 7, 25039.

(60) Bégu, S.; Pouëssel, A. A.; Lerner, D. A.; Tourné-Péteilh, C.; Devoisselle, J. M. Liposil, a promising composite material for drug storage and release. J. Controlled Release 2007, 118 (1), 1-6.

(61) Begu, S.; Girod, S.; Lerner, D. A.; Jardiller, N.; Tourne-Peteilh, C.; Devoisselle, J. M. Characterization of a phospholipid bilayer entrapped into non-porous silica nanospheres. J. Mater. Chem. 2004, 14 (8), 1316-1320.

(62) Steinberg, Y.; Schroeder, A.; Talmon, Y.; Schmidt, J.; Khalfin, R. L.; Cohen, Y.; Devoisselle, J.-M.; Begu, S.; Avnir, D. Triggered Release of Aqueous Content from Liposome-Derived Sol-Gel Nanocapsules. Langmuir 2007, 23 (24), 12024-12031.

(63) Askes, S. H. C.; Pomp, W.; Hopkins, S. L.; Kros, A.; Wu, S.; Schmidt, T.; Bonnet, S. Imaging Upconverting Polymersomes in Cancer Cells: Biocompatible Antioxidants Brighten Triplet-Triplet Annihilation Upconversion. Small 2016, 12 (40), 5579-5590.

(64) Marsh, D. Handbook of Lipid Bilayers, 2nd ed.; Taylor \& Francis Group: Boca Raton, FL, 2013; p 380, 483-484, 548-549.

(65) Askes, S. H. C.; Brodie, P.; Bruylants, G.; Bonnet, S. Temperature dependence of triplet-triplet annihilation upconversion in phospholipid membranes. J. Phys. Chem. B 2017, DOI: 10.1021/ acs.jpcb.6b10039.

(66) Wang, F.; Nimmo, S. L.; Cao, B.; Mao, C. Oxide formation on biological nanostructures via a structure-directing agent: towards an understanding of precise structural transcription. Chem. Sci. 2012, 3 (8), 2639-2645.

(67) Chen, S.; Hayakawa, S.; Shirosaki, Y.; Fujii, E.; Kawabata, K.; Tsuru, K.; Osaka, A. Sol-Gel Synthesis and Microstructure Analysis of Amino-Modified Hybrid Silica Nanoparticles from Aminopropyltriethoxysilane and Tetraethoxysilane. J. Am. Ceram. Soc. 2009, 92 (9), 2074-2082.

(68) van Blaaderen, A.; Vrij, A. Synthesis and Characterization of Monodisperse Colloidal Organo-silica Spheres. J. Colloid Interface Sci. 1993, 156 (1), 1-18.

(69) Askes, S. H. C.; Lopez Mora, N.; Harkes, R.; Koning, R. I.; Koster, B.; Schmidt, T.; Kros, A.; Bonnet, S. Imaging the lipid bilayer of giant unilamellar vesicles using red-to-blue light upconversion. Chem. Commun. 2015, 51 (44), 9137-9140.

(70) Wang, X.-d.; Stolwijk, J. A.; Lang, T.; Sperber, M.; Meier, R. J.; Wegener, J.; Wolfbeis, O. S. Ultra-Small, Highly Stable, and Sensitive Dual Nanosensors for Imaging Intracellular Oxygen and $\mathrm{pH}$ in Cytosol. J. Am. Chem. Soc. 2012, 134 (41), 17011-17014.

(71) Liu, Q.; Yang, T. S.; Feng, W.; Li, F. Y. Blue-Emissive Upconversion Nanoparticles for Low-Power-Excited Bioimaging in Vivo. J. Am. Chem. Soc. 2012, 134 (11), 5390-5397.

(72) Wohnhaas, C.; Mailander, V.; Droge, M.; Filatov, M. A.; Busko, D.; Avlasevich, Y.; Baluschev, S.; Miteva, T.; Landfester, K.; Turshatov, A. Triplet-Triplet Annihilation Upconversion Based Nanocapsules for Bioimaging Under Excitation by Red and Deep-Red Light. Macromol. Biosci. 2013, 13 (10), 1422-1430.

(73) Almgren, M. Migration and partitioning of pyrene and perylene between lipid vesicles in aqueous solution studied with a fluorescence stopped-flow technique. J. Am. Chem. Soc. 1980, 102 (27), 7882-7887.

(74) Kim, S. H.; Han, O. H.; Kim, J. K.; Lee, K. H. Multinuclear Solid-state NMR Investigation of Nanoporous Silica Prepared by Sol- gel Polymerization Using Sodium Silicate. Bull. Korean Chem. Soc. 2011, 32 (10), 3644-3649.

(75) Rånby, B.; Rabek, J. F. Singlet Oxygen Reactions with Organic Compounds \& Polymers. John Wiley \& Sons: New York, 1978.

(76) Carey, F. A.; Sundberg, R. J. Advanced Organic Chemistry, 5th ed.; Springer: New York, 2007; p 1322. 